\title{
Index of Subfactors and Statistics of Quantum Fields. I
}

\author{
Roberto Longo* \\ Dipartimento di Matematica, Università di Roma "Tor Vergata". Via Fontanile di Carcaricola, I-00133 \\ Roma, Italy.
}

\begin{abstract}
We identify the statistical dimension of a superselection sector in a local quantum field theory with the square root of the index of a localized endomorphism of the quasi-local $\mathrm{C}^{*}$-algebra that represents the sector. As a consequence in a two-dimensional theory the possible values of the statistical dimension below 2 are restricted to a given discrete set.
\end{abstract}

\section{Contents}

1. Introduction . . . . . . . . . . . . . . . . . . . . . 217

2. Index of Subfactors (Semifinite Case). . . . . . . . . . . . . 221

3. A Formula for the Canonical Endomorphism. . . . . . . . . . 223

4. Index of Subfactors (General Case) . . . . . . . . . . . . . 225

5. The Space of the Conditional Expectations . . . . . . . . . . . . . 228

6. Index and Compact Actions. . . . . . . . . . . . . . . . 232

7. Index and Parastatistics . . . . . . . . . . . . . . . . . 237

8. The Statistics of Low Dimensional Theories . . . . . . . . . . 243

9. Outlook . . . . . . . . . . . . . . . . . . . . . . . 246 References . . . . . . . . . . . . . . . . . 246

\section{Introduction}

In this paper we shall exhibit a natural connection between the statistics of local quantum fields and the index theory of subfactors. In particular the statistical dimension of a superselection sector [7] will appear as the square root of the index of an associated inclusion of von Neumann algebras [19]. The restriction on the possible values of the index [19] then imposes a corresponding restriction on the possible values of the statistical dimension. In particular for a two space-time dimensional quantum field theory the range of the statistical dimension consists of a discrete series and possibly of a continuous part.

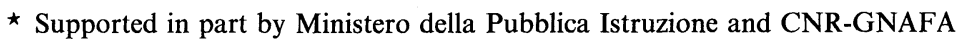


We now explain in more detail the ideas and the results in our investigation.

Index of subfactors and the joint modular structure. If one compares Jones index theory of subfactors [19] with the analysis of the joint modular structure of an inclusion of von Neumann algebras [21-24] one finds several analogies. The most evident one is that in both cases one starts with an inclusion of von Neumann algebras $N \subset M$ and builds up a canonical chain of von Neumann algebras $\cdots \subset M_{n} \subset M_{n+1} \subset \cdots$; this construction is initially (and step by step) given by the same formula

$$
M_{1}=J_{M} N^{\prime} J_{M},
$$

where $J_{M}$ is the modular conjugation of $L^{2}(M)$. In [19] however $M$ is a finite factor and one constructs the chain by using trace of $M_{n}$ and represents at each step the von Neumann algebras on the different Hilbert spaces $L^{2}\left(M_{n}\right)$.

On the other hand in [21] $M$ is properly infinite, no privileged state exists, but one may choose a common cyclic separating vector $\Omega$ for $N$ and $M$ and associate with $\Omega$ a cononical endomorphism $\gamma_{\Omega}: M \rightarrow N$ of $M$ into $N$. Setting $M_{n} \equiv \gamma_{\Omega}^{n}(M)$ one has indeed

$$
M_{1}=\gamma^{-1}(M)=J_{M} N J_{M}^{\prime} .
$$

The arbitrariness in the choice of $\Omega$ is compensated by the fact the all the construction is made on the initial Hilbert space and by the uniqueness (RadonNikodym property) of the canonical endomorphism. It seems that these constructions are extreme cases of the same method.

As pointed out to us by $\mathrm{V}$. Jones the index of an inclusion of $I I_{1}$ factors $N \subset M$ may be defined as the scaling factor $\lambda \in \mathbf{R}$,

$$
\tau \cdot \gamma_{\Omega}=\lambda \tau
$$

of the trace of $M \otimes F$ by the canonical endomorphism $\gamma_{\Omega}: M \otimes F \rightarrow N \otimes F$ ( $F$ a type $I_{\infty}$ factor).

In fact we shall show that, by using Takesaki duality and the RadonNikodym property of the canonical endomorphism, one may define an index for arbitrary inclusions of factors.

A definition of the index for infinite factors already existed due to Haagerup and Kosaki [18]. Our definition agrees with that one, but our approach appears natural for our purposes.

In particular Jones theorem $[19,18]$ restricts the values of the index $I$ to

$$
I \in\left\{4 \cos ^{2} \frac{\pi}{n}, n=3,4, \ldots\right\} \cup[4, \infty] .
$$

The index of an inclusion of factors $N \subset M$ depends on the choice of a normal faithful conditional expectation $\varepsilon$ of $M$ onto $N$. An interpretation of this index $I=\operatorname{Ind}_{\varepsilon}(N, M)$ as the square of a dimension requires however that $I^{1 / 2}$ must satisfy an additive property, namely if $\left\{p_{i}\right\}$ is a partition of the unit by projections of $N^{\prime} \cap M$ then

$$
I^{1 / 2}=\sum_{i} I_{i}^{1 / 2}
$$

where $I_{i}=\operatorname{Ind}_{\varepsilon_{i}}\left[N_{p_{i}}, M_{p_{i}}\right]$ is the index of $N_{p_{i}} \subset M_{p_{i}}$ induced by $\varepsilon$. 
It turns out that this additive requirement fixes $\varepsilon$ uniquely, assuming that $I$ is finite, and in fact $\varepsilon$ will appear as the unique conditional expectation that minimizes the index (the space of the conditional expectations will be compact and the function $\varepsilon \rightarrow \operatorname{Ind}_{\varepsilon}(N, M)$ continuous and strictly convex on tracial expectations, thus the minimum is taken at a unique point). A different characterization of this expectation has been independently obtained in [17].

A natural and useful point in our analysis is the extension to infinite factors of an inquality due to Pimsner and Popa [29] in the finite case, namely $\lambda=\operatorname{Ind}_{\varepsilon}(N, M)^{-1}$ is the best constant such that

$$
\varepsilon(x) \geqq \lambda x
$$

for all $x \in M^{+1}$.

A further result consists of the connection between the index of an inclusion of factors $N \subset M$ and the index of the fixed point algebra inclusion $N^{\alpha} \subset M^{\alpha}$ under a dominant action of a compact group.

In fact our analysis originated in our previous study of the modular structure of a crossed product by a group dual [27].

As a corollary we find examples of subfactors of a factor $M$ with trivial relative commutant and index $n^{2}, n \in \mathrm{N}$, that are associated with a prime action of a compact group $G$ on $M$ and an irreducible representation of $G$. By our results, also two dimensional quantum field models furnish examples of subfactors with trivial relative commutant a non-integral index. Their analysis is presently under investigation.

The Index of a Superselection Sector. Beside the usual Fermi-Bose particle statistics, Quantum Mechanics provides a theoretical description of parastatistics [15] where the symmetry of the $n$-particle wave function is realized by a representation of the permutation group $\mathbf{P}_{n}$ of dimension possibly greater than one (the isotopic identification of proton and neutron or quarks are examples cf. [7]).

In [7] Doplicher, Haag and Roberts described the structure of the superselection sectors in a local Quantum Field Theory. They showed that a superselection sector corresponds to (an equivalence class of) a localized endomorphism $\varrho$ of the quasi-local $C^{*}$-algebra $\mathscr{A}=\cup \mathscr{A}(\mathcal{O})^{-}$.

The statistics of the sector $\varrho$ is then completely described by an associated statistics parameter $\lambda_{\varrho}$. If $\varrho$ is irreducible then $\lambda_{\varrho}$ is a scalar and its possible values are restricted to

$$
\lambda_{\varrho} \in\left\{0, \pm, 1, \pm \frac{1}{2} \pm \frac{1}{3} \cdots\right\}
$$

In general $d(\varrho) \equiv\left|\lambda_{\varrho}\right|^{-1}$ is an integer, the statistical dimension of $\varrho$.

If one compares this structure with Jones work, one discovers a similarity both in the results and in the methods.

\footnotetext{
${ }^{1}$ This inequality has also been considered in [34] and in a recent announcement [16]
} 
We shall show that in general

$$
d(\varrho)=\operatorname{Ind}[\varrho(\mathscr{A}(\mathcal{O})), \mathscr{A}(\mathcal{O})]^{1 / 2},
$$

where $\mathcal{O}$ is a sufficiently large region of the Minkowski space so that $\varrho$ is localized in a double cone within $\mathcal{O}$ and Ind denotes the minimal index as discussed above.

Of this result, we shall give two proofs that will cover different needs. The first proof assumes the existence of a field algebra and a gauge group, with normal commutation relations, and has the merit of a transparent revealing of the underlying structure and of the ultimate reason for the validity of the result. Furthermore these hypotheses have been recently derived from first principles [9].

The second proof only uses observables and relies on the analysis in [7] and Theorem 4.1. It has the merit of being the starting point of a further analysis where the field algebra is unknown. In fact the validity of the results in [7] relies on the fact that the Minkowski space has space-time dimension $d>2$. Our result also extends to the more general setting of charges localized in space-like cones [4] provided $d>3$.

The Statistics in Two-Dimensional Theories. It has long been realized that in two space-time dimensions the statistics of local fields does not always reduce to ordinary Bose and Fermi parastatistics but one has the appearance of exotic statistics, a fact tied up to the possibility of abnormal field commutation relations, see $[14,33]$ and references therein.

Recently this subject is receiving interest also because of its connections with such different topics as soliton quantization, conformal field theory and superstring theory, and polynomial invariants for knots and links, see [14]. It is then natural to extend our previous analysis to this case.

In two dimensions however the full analysis in [7] is not applicable. The geometric reason is that the space-like complement of a point is no longer a connected region, but one has to care about the left and the right space-like complement. It is widely known to experts that this amounts to replacing the permutation group by the braid group, a fact that shall play an implicit role in our analysis.

Assuming that the sector $\varrho$ admits a conjugate sector $\bar{\varrho}$, we shall see that the statistical dimension $d(\varrho)=\left|\lambda_{\varrho}\right|^{-1}$ is still definable and as above for large $\mathcal{O}$,

$$
d(\varrho)=\operatorname{Ind}[\varrho(\mathscr{A}(\mathcal{O})), \mathscr{A}(\mathcal{O})]^{1 / 2},
$$

where the index is relative to a standard left inverse of $\varrho$ that, under a regularity condition, turns out to be the minimal index.

One immediately deduces that

$$
d(\varrho) \in\left\{2 \cos \frac{\pi}{n}, n=3,4 \ldots\right\} \cup[2, \infty] .
$$

The continuous part $[2, \infty]$ in the range of $d(\varrho)$ is probably not optimal, being related to the open problem on the index values of subfactors with trivial relative commutant in the injective case, see the Outlook.

There is a natural continuation of our work by an analysis of the braid group 
representations that appear, by relating $d(\varrho)$ with the central charge in conformal quantum field theory (see [13]) and others that we discuss in the Outlook.

Our results have been announced in [26].

\section{Index of Subfactors (Semifinite Case)}

We begin here a study of the index of an inclusion of von Neumann algebras $N \subset M$; without further discussion we restrict ourselves to the case of von Neumann algebras with separable preduals, although this restriction is not needed for the essential part of this paper.

Denote by End $(M, N)$ the semigroup of the endomorphisms of $M$ mapping $N$ into $N$ and by $\operatorname{Aut}(M, N)$ the corresponding automorphism subgroup.

If $\tau$ is a normal faithful semifinite trace of $M$ and $\alpha \in \operatorname{End}(M)(\equiv \operatorname{End}(M, C)), \tau \cdot \alpha$ is a trace on $M$. The modulus of $\alpha$ is the Random-Nikodym derivative

$$
\bmod (\alpha)=\bmod _{\tau}(\alpha)=(d \tau \cdot \alpha: d \tau)
$$

namely $\bmod (\alpha)$ is the unique positive operator $h$ affiliated with the center $Z(M)$ of $M$ such that

$$
\tau(\alpha(x))=\tau(h x), \quad x \in M_{+}
$$

(if $\alpha$ is an endomorphism $h$ is allowed to be $\infty$ on a subspace).

We shall consider the subgroup $G(\tau)$ of $\operatorname{Aut}(M, N)$,

$$
G(\tau) \equiv\{\alpha \in \operatorname{Aut}(M, N), \bmod (\alpha) \in(0, \infty)\},
$$

and shall say that $\tau$ is centrally ergodic if $G(\tau)$ acts ergodically on $Z(M)$.

Assuming that $N$ and $M$ are properly infinite there exists a normal faithful state $\omega \in M_{*}$ represented (in the GNS construction) by a vector $\Omega \in L^{2}(M)$ cyclic and separating for both $M$ and $N$ (briefly a cyclic state for $N$ and $M$ ) [10].

The associated canonical endomorphism $\gamma_{\omega}: M \rightarrow N$ is the endomorphism $\gamma_{\omega} \in \operatorname{End}(M, N)$ given by

$$
\gamma_{\omega}(x)=\Gamma x \Gamma^{*}, \quad x \in M,
$$

where $\Gamma=J_{N} J_{M}$ with $J_{N}$ and $J_{M}$ the modular conjugations of $N$ and $M$ respectively with respect to $\Omega$ [21].

Lemma 2.1. $\bmod \left(\gamma_{\omega}\right)$ depends only on $M, N$ and $\tau$ and not on $\omega$.

Proof. If $\varphi$ is another cyclic state for $N, M$, there exists a unitary $u \in N$ such that [22]

$$
\gamma_{\varphi}=u \gamma_{\omega}(\cdot) u^{*}
$$

hence

$$
\tau\left(\gamma_{\varphi}(x)\right)=\tau\left(u \gamma_{\omega}(x) u^{*}\right)=\tau\left(\gamma_{\omega}(x)\right)=\tau\left(\bmod \left(\gamma_{\omega}\right) x\right), \quad x \in M^{+},
$$

thus $\bmod \left(\gamma_{\varphi}\right)=\bmod \left(\gamma_{\omega}\right)$.

A trace $\tau$ of $M$ such that $\bmod _{\tau}(\gamma)$ is a scalar will be called a scalar trace.

Proposition 2.2. If $\tau$ is centrally ergodic then $\tau$ is a scalar trace and $\bmod _{\tau}(\gamma) \geqq 1$. 
Proof. Let $h=\bmod _{\tau}\left(\gamma_{\omega}\right)$ and $\alpha \in G(\tau)$. Since

we have by Lemma 2.1

$$
\alpha \cdot \gamma_{\varphi} \cdot \alpha^{-1}=\gamma_{\varphi \cdot \alpha}
$$

$$
\tau\left(\left(\alpha \cdot \gamma_{\omega} \cdot \alpha^{-1}\right)(x)\right)=\tau\left(\gamma_{\varphi \cdot \alpha}(x)\right)=\tau(h x), \quad x \in M^{+} .
$$

On the other hand

$$
\tau\left(\left(\alpha \cdot \gamma_{\omega} \cdot \alpha^{-1}(x)\right)=\bmod (\alpha) \tau\left(\gamma_{\omega}\left(\alpha^{-1}(x)\right)=\bmod (\alpha) \tau\left(h \alpha^{-1}(x)\right)=\tau(\alpha(h) x),\right.\right.
$$

thus $\alpha(h)=h$ and $h \in[0, \infty]$ by the ergodicity of $G(\tau)$.

If $h<1$ choose $x \in M^{+}$with $0<\tau(x)<\infty$; then $\tau\left(\gamma_{\omega}^{n}(x)\right)=h^{n} \tau(x) \rightarrow 0$. On the other hand $\gamma_{\omega}^{n}$ converges pointwise weakly to a faithful conditional expectation $\varepsilon$ [21]. By the lower semicontinuity of $\tau$ we have $\tau(\varepsilon(x)) \leqq \lim _{n} \tau\left(\gamma_{\omega}^{n}(x)\right)=0$, thus $x=0$ because $\tau$ is faithful. This contradiction shows that $h \geqq 1$.

By Lemma 2.1 we may define the index of $N$ in $M$ as the operator (scalar in the above case)

$$
\operatorname{Ind}_{\tau}(N, M) \equiv \bmod _{\tau}\left(\gamma_{\omega}\right)
$$

that we shall later relate to the Jones index.

Remark. If $M$ is a $I I_{\infty}$ factor the fundamental group of $M$ is $\{\bmod (\alpha), \alpha \in \operatorname{Aut}(M)\}$. One can also consider the fundamental semigroup of $M,\{\bmod (\alpha), \alpha \in \operatorname{End}(M)\}$; the index value for the subfactors of $M$ thus appears as a sub-semigroup of the latter where one considers only canonical endomorphisms.

Proposition 2.3. If $\tau$ is a scalar trace and $\operatorname{Ind}_{\tau}(M, N)<\infty$, there exists a type $I_{\infty}$ subfactor $F$ of $N$ such that $F^{\prime} \cap M$ is a finite von Neumann algebra. In particular there exists a normal faithful conditional expectational expectation of $M$ onto $N$.

Proof. If $\operatorname{Ind}_{\tau}(N, M)<\infty \tau \cdot \gamma_{\varphi}$ is a semifinite trace on $M$, thus $\tau \mid \gamma_{\varphi}(M)$ is semifinite. Choose $F$ a type $I_{\infty}$ subfactor of $\gamma_{\varphi}(M)$ such that $A \equiv F^{\prime} \cap \gamma_{\varphi}(M)$ is finite and set $B=F^{\prime} \cap M$. Then we have the tensor product decompositions $M=B \otimes F$ and $\gamma_{\varphi}(M)=A \otimes F$, where $\tau=\tau^{\prime} \otimes \operatorname{Tr}$ with $\tau^{\prime}$ a trace of $B$. Since $\tau^{\prime} \mid A$ is semifinite and $A$ is finite, $\tau^{\prime}$ is finite on $A$, i.e. $\tau^{\prime}(1)<\infty$, thus also $B$ is finite.

Example. Let $M=M_{0} \otimes F_{n}, N=M_{0} \otimes 1$, with $F_{n}$ a type $I_{n}$ factor and $M_{0}$ a properly infinite von Neumann algebra. Then $\gamma_{\varphi}: M \rightarrow N$ is inner and $H\left(\gamma_{\varphi}\right)=\left\{v \in M \mid \gamma_{\varphi}(x) v=\right.$ $v x, x \in M\}$ is a Hilbert space in $M$ of dimension $n^{2}$ [22].

Thus if $\tau$ is a trace of $M$

$$
\tau\left(\gamma_{\varphi}(x)\right)=\sum_{i=1}^{n^{2}} \tau\left(v_{i} x v_{i}^{*}\right)=\sum_{i=1}^{n^{2}} \tau\left(x v_{i}^{*} v_{i}\right)=n^{2} \tau(x)
$$

and $\operatorname{Ind}_{\tau}(N, M)=n^{2}$.

Proposition 2.4. Denate by $M_{1} \equiv J_{M} N^{\prime} J_{M}$ the extension of $M$ by $N$. If $\tau$ is a scalar trace on $M$ and $h \equiv \operatorname{Ind}_{\tau}(N, M)<\infty$, there exists a canonical trace on $\tilde{\tau}$ on $M_{1}$ that extends $\tau$. This $\tilde{\tau}$ is a scalar trace for $M \subset M_{1}$ and $\operatorname{Ind}_{\tau}\left(M, M_{1}\right)=h$.

Proof. By choosing a cyclic state $\omega$ for $N$ and $M$ the unitary $\Gamma=J_{N} J_{M}$ implements a canonical endomorphism $\gamma_{\omega}: M_{1} \rightarrow M$ (because $J_{M_{1}}=J_{M} J_{N} J_{M}$ ), thus $\gamma_{\omega}$ restricts 
to the canonical endomorphism of $M$ into $N$. If $h^{-1}$ is bounded we may put

$$
\tilde{\tau}(x) \equiv \tau\left(\gamma\left(h^{-1} x\right)\right), \quad x \in M .
$$

Thus $\tilde{\tau}$ is a trace on $M_{1}$ and if $x \in M$

$$
\tilde{\tau}(x)=\tau\left(\gamma\left(h^{-1} x\right)\right)=\tau\left(h h^{-1} x\right)=\tau(x) .
$$

To check that $\tilde{\tau}$ is a scalar trace for $M \subset M_{1}$, notice that since $\gamma$ is a canonical endomorphism of $M_{1}$ into $M$, we have

$$
\tilde{\tau}(\gamma(x))=\tau\left(\gamma\left(h^{-1} \gamma(x)\right)=\tau\left(h h^{-1} \gamma(x)\right)=\tau(\gamma(x))=\tau(h x), \quad x \in M,\right.
$$

that concludes the proof.

\section{A Formula for the Canonical Endomorphism}

Let $N \subset M$ be an inclusion of Neumann algebras on a Hilbert space $\mathscr{H}$ and $\Omega$ a common cyclic separating vector for $N$ and $M$. Suppose there exists a normal faithful conditional expectation $\varepsilon$ of $M$ onto $N$; we wish to describe the canonical endomorphism $\gamma_{\Omega}: M \rightarrow N$ in this case.

Let $\omega=(\cdot \Omega, \Omega) \in M_{*}, \varphi=\omega \cdot \varepsilon \in M_{*}^{+}$and $\xi \in L^{2}(M)_{+}$the positive vector representative of $\varphi$.

The projection

$$
e=[N \xi] \in N^{\prime}
$$

commutes with $J_{M}$. Since $\xi$ is separating for $N$, the homomorphism

$$
\phi: N \rightarrow N e, \quad x \rightarrow x e
$$

is an isomorphism. Let

$$
V: \mathscr{H}=L^{2}(N, \Omega) \rightarrow e \mathscr{H}=\left(N_{e}, \xi\right)
$$

the unitary standard implementation of $\phi$ that we regard as the isometry of $\mathscr{H}$ with final projection $e$ such that

$$
\begin{gathered}
V x V^{*}=\phi(x)=x e, \quad x \in N \\
V \Omega \in L^{2}(\mathrm{Ne}, \xi)_{+} \cdot
\end{gathered}
$$

The modular conjugation of $L^{2}(N e, \xi)$ is

$$
J_{N_{e}}=J_{M}^{\xi} \mid e \mathscr{H},
$$

therefore

$$
J_{N} \equiv J_{N}^{\Omega}=V^{*} J_{M}^{\xi} V
$$

and

$$
\Gamma_{\Omega} \equiv J_{N}^{\Omega} J_{M}^{\Omega}=V^{*} J_{M}^{\xi} V J_{M}^{\xi}
$$

or

$$
\Gamma=V^{*} J_{M} V J_{M}
$$

because $J_{M}^{\Omega}=J_{M}^{\xi}$ as $\xi \in L^{2}(M, \Omega)_{+}$. 
Proposition 3.1. With the above notations,

$$
\Gamma=V^{*} W,
$$

where $V, W$ are isometries with final projection $e \in M, V \in N^{\prime}$ and $W=J_{M} V J_{M} \in M_{1}$, $\left(M_{1} \equiv J_{M} N^{\prime} J_{M}\right)$.

Proof. Immediate by the above considerations.

Remark. The above proposition remains true if $\Gamma=J_{N} J_{M}$, where $J_{N}$ and $J_{M}$ are modular conjugations of $N$ and $M$ with respect to different vectors. In fact any other modular conjugation $J_{N}^{\prime}$ of $N$ is given by $J_{N}^{\prime}=u J_{N} u^{*}$ with $u$ a unitary of $N$.

Let $A \subset B$ be an inclusion of finite von Neumann algebras, $\xi$ a cyclic (thus separating) trace unit vector for $B$ and $B_{1}=J_{B} A^{\prime} J_{B}$ the extension of $B$ by $A$.

Let $\tau_{B}=(\cdot \xi, \xi)$ be the corresponding tracial state of $B$. By considering the inclusion

$$
N \equiv A \otimes F \subset M \equiv B \otimes F,
$$

where $F$ is a type $I_{\infty}$ factor, we see by Proposition 2.4 that $\tau_{B} \otimes \operatorname{Tr}$ extends to a trace $\tau \equiv \tau_{B_{1}} \otimes \operatorname{Tr}$ of $M_{1} \equiv B_{1} \otimes F$, where $\tau_{B_{1}}$ is a trace of $B_{1}$. Put $e_{0}=[A \xi] \in B_{1}$.

In the case where $A$ and $B$ are factors the next proposition shows that $\operatorname{Ind}_{\tau}(N, M)$ coincides with the $I I_{1}$-index of $A$ in $B$ [19] and follows a comment by V. Jones.

Proposition 3.2. If $G(\tau)$ acts ergodically on $Z(N)$ then

$$
\operatorname{Ind}_{\tau}(N, M)=\tau_{B_{1}}\left(e_{0}\right)^{-1} \text {. }
$$

Proof. Let $\Omega=\xi \otimes \eta$ where $\eta$ is a cyclic separating vector for $F$. We want to compute

$$
\left(\tau_{B} \otimes \operatorname{Tr}\right)(\gamma(x)) \quad x \in M
$$

where $\gamma: M \rightarrow N$ is a canonical endomorphism. By Proposition 3.1,

$$
\gamma=\phi^{-1} \cdot \psi
$$

where $\psi: x \rightarrow W x W^{*}$ is an isomorphism of $M$ into $N_{e}, e=e_{0} \otimes 1$, and $\phi$ is the reduction of $N$ onto $N_{e}$;

$$
\begin{aligned}
\tau(\gamma(x)) & =\tau_{B} \otimes \operatorname{Tr}(\gamma(x))=\tau_{B} \otimes \operatorname{Tr}\left(\phi^{-1}(\psi(x))\right) \\
& =\tau_{A} \otimes \operatorname{Tr}\left(\phi^{-1}(\psi(x))\right), \quad\left(\tau_{A}=\tau_{B} \mid A\right) \\
\tau_{A e_{0}} & \otimes \operatorname{Tr}(\psi(x)),
\end{aligned}
$$

where $\tau_{A e_{0}}$ is the trace of $A e_{0}$ given by

$$
\tau_{A e_{0}}\left(y e_{0}\right)=\tau_{A}(y), \quad y \in A .
$$

Let $\varepsilon$ be the conditional expectation of $B_{1}$ onto $B$ preserving $\tau_{B_{1}}$. If $\alpha \in \mathrm{G}\left(\tau_{B} \otimes \mathrm{Tr}\right)$ it extends to an automorphism $\alpha$ of $B_{1} \otimes F$ that commutes with $\varepsilon \otimes$ id and fixes $e$ by canonicity.

Moreover as in [19, Proposition 3.1.7] if $x \in B$ and $\varepsilon_{A}$ is the $\tau_{B_{1}}$-preserving conditional expectation of $B_{1}$ onto $A$ we have

$$
\tau_{B_{1}}\left(x e_{0}\right)=\tau_{B_{1}}\left(e_{0} x e_{0}\right)=\tau_{B_{1}}\left(\varepsilon_{A}(x) e_{0}\right)=\tau_{B_{1}}\left(x \varepsilon_{A}\left(e_{0}\right)\right)
$$


that shows $\varepsilon\left(e_{0}\right)=\varepsilon_{A}\left(e_{0}\right) \in Z(A)$ (because $\left.e_{0} \in A^{\prime} \cap B_{1}\right)$. Since $\varepsilon\left(e_{0}\right)$ is fixed by $G\left(\tau_{B_{1}}\right)$, it must be a scalar $\varepsilon\left(e_{0}\right)=\tau_{B_{1}}\left(\varepsilon\left(e_{0}\right)\right)=\tau_{B_{1}}\left(e_{0}\right)$.

We have

$$
\tau_{B_{1}}\left(y e_{0}\right)=\tau_{B_{1}}\left(y \varepsilon\left(e_{0}\right)\right)=\tau_{B_{1}}\left(e_{0}\right) \tau_{B_{1}}(y), \quad y \in A
$$

that shows the equality

$$
\tau_{A e_{0}}=\left.\tau_{B_{1}}\left(e_{0}\right)^{-1} \tau_{B_{1}}\right|_{A e_{0}}
$$

It follows that if $x \in M^{+}$,

$$
\begin{aligned}
\tau(\gamma(x)) & =\tau_{A_{0}} \otimes \operatorname{Tr}(\psi(x)) \\
& =\tau_{B_{1}}\left(e_{0}\right)^{-1} \tau_{B_{1}} \otimes \operatorname{Tr}\left(W x W^{*}\right) \\
& =\tau_{B_{1}}\left(e_{0}\right)^{-1} \tau_{B_{1}} \otimes \operatorname{Tr}\left(x W^{*} W\right) \\
& =\tau_{B_{1}}\left(e_{0}\right)^{-1} \tau_{B_{1}} \otimes \operatorname{Tr}(x)=\tau_{B_{1}}\left(e_{0}\right)^{-1} \tau(x)
\end{aligned}
$$

that implies

$$
\bmod _{\tau}(\gamma)=\tau_{B_{1}}\left(e_{0}\right)^{-1}
$$

As a corollary we have in this setting an inequality of [29] that we shall generalize later.

Corollary 3.3. Let $N \subset M$ be an inclusion of properly infinite von Neumann algebras and $\tau$ a centrally ergodic trace for $N \subset M$. Then $\lambda=\operatorname{Ind}_{\tau}(N, M)^{-1}$ is the best constant such that

$$
\varepsilon(x) \geqq \lambda x, \quad x \in M^{+},
$$

where $\varepsilon$ is the $\tau$-preserving conditional expectation of $M$ onto $N$.

Proof. Let $\gamma: M \rightarrow N$ be a canonical endomorphism, then $M$ is the extension of $N$ by $\gamma(N)$. By using this fact and Proposition 2.4, the proof follows the same argument in [29] because $\varepsilon(e)=\lambda$, where $e$ is the Jones projection as in the proof of Lemma 3.2.

\section{Index of Subfactors (General Case)}

Let $N \subset M$ be an inclusion of factors with separable preduals. In order to define the index of $N$ in $M$ we may assume that $N$ and $M$ are infinite, otherwise replacing $N \subset M$ by $N \otimes F \subset M \otimes F$, with $F$ a type $I_{\infty}$ factor.

We assume the existence of a normal faithful conditional expectation $\varepsilon$ of $M$ onto $N$ and we denote by $C(M, N)$ the set of all normal conditional expectations of $M$ onto $N$.

Given a faithful normal state $\varphi_{0} \in N_{*}$ the modular group $\sigma^{\varphi}$ of the state $\varphi=\varphi_{0}{ }^{\circ} \varepsilon$ leaves $N$ globally stable and we have an inclusion of semifinite algebras [31]

$$
\tilde{N} \subset \tilde{M},
$$

where $\tilde{N}=N \times{ }_{\sigma^{\varphi_{0}}} \mathbf{R}, \tilde{M}=M \times{ }_{\sigma^{\phi}} \mathbf{R}$ are the crossed product von Neumann algebras. The inclusion $\tilde{N} \subset \tilde{M}$ does not depend on $\varphi_{0}$, up to isomorphism, because if $\psi_{0} \in N_{*}$ 
is another faithful state and $\psi=\psi_{0} \cdot \varepsilon \in M_{*}$, then the Connes cocycle

$$
(D \psi: D \varphi)_{t}=\left(D \psi_{0}: D \varphi_{0}\right)_{t}
$$

belongs to $N[6]$.

The canonical trace $\tau$ of $\tilde{M}$ is relatively invariant under the dual action $\Theta: \mathbf{R} \rightarrow \operatorname{Aut}(\tilde{M}, \tilde{N})$,

$$
\tau \cdot \Theta_{t}=e^{-t} \tau, \quad t \in \mathbf{R} .
$$

Since $\Theta_{t} \mid Z(\tilde{M})$ is ergodic, $\tau$ is a scalar trace by Proposition 2.2 and we define

$$
\operatorname{Ind}_{\varepsilon}(N, M)=\operatorname{Ind}_{\tau}(\tilde{N}, \tilde{M}) \text {. }
$$

An immediate verification shows that if $N \subset M$ are semifinite, and $\tau_{0}$ is a trace on $M$ then

$$
\operatorname{Ind}_{\tau_{0}}(N, M)=\operatorname{Ind}_{\varepsilon_{0}}(N, M),
$$

where $\varepsilon_{0} \in C(M, N)$ is the $\tau_{0}$-preserving conditional expectation that exists if $\operatorname{Ind}_{\tau_{0}}(N, M)$ is finite by Proposition 2.3.

Let $\tilde{\varphi}$ be the dual weight of $\varphi$ on $\tilde{M}$,

$$
\tilde{\varphi}(x)=\varphi\left(\int \Theta_{t}(x) d t\right), \quad x \in \tilde{M}^{+} .
$$

Then $\tilde{\varphi} \mid \tilde{N}$ equals $\tilde{\varphi}_{0}$, the dual weight of $\varphi_{0}$, and in particular it is semifinite on $N$. The modular group $\sigma^{\tilde{\varphi}}$ of $\tilde{\varphi}$ is given by

$$
\sigma^{\tilde{\varphi}} \mid M=\sigma^{\varphi}, \quad \sigma_{t}^{\varphi}\left(U_{s}\right)=U_{s}, \quad t, s \in \mathbf{R},
$$

where the $U_{s}$ are the usual unitaries in $\tilde{M}$; in particular $\left.\sigma^{\tilde{\varphi}}\right|_{\tilde{N}}=\sigma^{\tilde{\varphi}_{0}}$ and by Takesaki criterium [32] there exists a faithful expectation $\tilde{\varepsilon} \in C(\tilde{M}, \tilde{N})$ that preserves $\tilde{\varphi}$; it follows that

$$
\tilde{\varepsilon} \mid M=\varepsilon, \quad \tilde{\varepsilon}\left(U_{s}\right)=U_{s} .
$$

Let $K$ be the infinitesimal generator of $U$ and $H=e^{-K}$, thus $H$ is a positive operator affiliated to $\tilde{N}$ and

It follows that

$$
\tau=\tilde{\varphi}(H \cdot)
$$

$$
\tau(\tilde{\varepsilon}(x))=\tilde{\varphi}(H \tilde{\varepsilon}(x))=\tilde{\varphi}(\tilde{\varepsilon}(H x))=\tilde{\varphi}(H x)=\tau(x), \quad x \in M^{+},
$$

where the equalities are justified by the spectral theorem; in other words $\tilde{\varepsilon}$ is the $\tau$-preserving conditional expectation of $\tilde{M}$ onto $\tilde{N}$.

We are now ready to extend the Pimsner-Popa inequality [29] to the general case.

Theorem 4.1. Let $N \subset M$ be an inclusion of infinite-dimensional factors, $\varepsilon \in C(M, N)$ a faithful conditional expectation and set $\lambda=\operatorname{Ind}_{\varepsilon}(N, M)^{-1}$. Then $\lambda$ is the best constant such that

$$
\varepsilon(x) \geqq \lambda x, \quad x \in M^{+} .
$$

Proof. We may assume that $M$ is properly infinite. Following the previous 
notations the extension $\tilde{\varepsilon}$ of $\varepsilon$ satisfies

$$
\tilde{\varepsilon}(x) \geqq \lambda x, \quad x \in \tilde{M}^{+},
$$

by Corollary 3.3 (because $\tilde{M}$ is semifinite), hence $\varepsilon=\tilde{\varepsilon} \mid M$ also satisfies this inequality. To show that $\lambda$ is the best constant consider the bidual weight $\tilde{\varphi}$ on $\tilde{M} \times{ }_{\theta} \mathbf{R}$. Then $\tilde{\varepsilon}$ extends again by Takesaki criterium to a conditional expectation $\tilde{\tilde{\varepsilon}}$ of $\tilde{M} \times{ }_{\theta} \mathbf{R}$ onto $\tilde{N} \times{ }_{\theta} \mathbf{R}$ that preserves $\tilde{\tilde{\varphi}}$. Since under the isomorphism of $\tilde{M} \times{ }_{\theta} \mathbf{R}$ with $M \otimes F, \tilde{\tilde{\varphi}}$ is mapped to $\varphi \otimes \operatorname{Tr}$ and $\tilde{N} \times{ }_{\Theta} \mathbf{R}$ onto $N \otimes F$, we have that $\tilde{\tilde{\varepsilon}}$ is mapped to $\varepsilon \otimes$ id (by the $\tilde{\tilde{\varphi}}$-invariance). It follows by the proof of Corollary 3.3 that there exists a projection $e \in \tilde{M} \subset \tilde{M} \times{ }_{\otimes} \mathbf{R} \simeq M \otimes F$ such that

$$
(\varepsilon \otimes \mathrm{id})(e)=\lambda \text {. }
$$

Now if we choose a type $I_{\infty}$ subfactor $F_{1}$ of $N$ with infinite relative commutant the tensor product decomposition

$$
M \simeq\left(F_{1}^{\prime} \cap M\right) \otimes F_{1}
$$

gives an isomorphism of $N \subset M$ with $N \otimes F_{1} \subset M \otimes F_{1}$ that maps $\varepsilon$ to $\varepsilon \otimes$ id, hence there exists a projection $p \in M$ with $\varepsilon(p)=\lambda$ and $\lambda$ is the best constant such that $\varepsilon(x) \geqq \lambda x, x \in M^{+}$.

Remark. If $M$ is finite dimensional, $\operatorname{Ind}_{\varepsilon}(M, N)^{-1}$ is not the best constant in the inequality of Theorem 4.1. For example if $M=B\left(\mathbf{C}^{n}\right), N=\mathbf{C}$ and $\varepsilon$ is the normalized trace, then $\operatorname{Ind}_{\varepsilon}(M, N)=n^{2}$ but the best constant is $1 / n$. However $\lambda$ is always the best constant such that $\varepsilon-\lambda \cdot$ id is completely positive.

Corollary 4.2. Let $N \subset M$ be an inclusion of factors and $\varepsilon \in C(M, N)$ a faithful conditional expectation. Then $\operatorname{Ind}_{\varepsilon}(N, M)$ coincides with the Kosaki index [18].

Proof. By Theorem 4.1 $\lambda=\operatorname{Ind}_{\varepsilon}(N, M)^{-1}$ is the best constant that satisfies the inequality $\varepsilon(x) \geqq \lambda x, x \in M_{+}$. But the same proof of [29, Proposition 21] shows that the inverse of the Kosaki index is such a best constant by applying [18, Lemma 3.1].

Corollary 4.3. Let $N \subset M$ be an inclusion of factors and $\varepsilon \in C(M, N)$ a faithful expectation. Then $\operatorname{Ind}_{\varepsilon}(N, M)$ belongs to $\left\{4 \cos ^{2} \pi / n, n=3,4 \ldots\right\} \cup[4, \infty]$.

Proof. Immediate by Corollary 4.2 and the extension of Jones theorem to infinite factors [18].

Corollary 4.4. Let $N \subset M, \varepsilon$ and $\lambda$ be as in Theorem 4.1. Then

$$
\lambda=\sup \left\{t \geqq 0,\|\varepsilon(x)\| \geqq t\|x\|, x \in M_{+}\right\} .
$$

Proof. If $x \in M_{+}$then $\varepsilon(x) \geqq \lambda x$, thus $\|\varepsilon(x)\| \geqq \lambda\|x\|$. On the other hand there exists a projection $e \in M$ with $\varepsilon(e)=\lambda$, thus $\|\varepsilon(e)\|=\lambda=\lambda\|e\|$ and $\lambda$ is the best constant for that inequality.

Proposition 4.5. If $N \subset N_{1} \subset M$ are factors, $\varepsilon_{1} \in C\left(M, N_{1}\right), \varepsilon_{2} \in C\left(N_{1}, N\right)$ are faithful then

with $\varepsilon=\varepsilon_{2} \cdot \varepsilon_{1}$.

$$
\operatorname{Ind}_{\varepsilon}(M, N)=\operatorname{Ind}_{\varepsilon_{1}}\left(M, N_{1}\right) \operatorname{Ind}_{\varepsilon_{2}}\left(N_{1}, N\right)
$$


Proof. This follows immediately by the chain rule for the canonical endomorphism $\left(\gamma_{\omega}: \tilde{M} \rightarrow \tilde{N}\right.$ is the product of $\gamma_{\omega}: \tilde{M} \rightarrow \tilde{N}_{1}$ and $\left.\gamma_{\omega}: \tilde{N}_{1} \rightarrow \tilde{N}\right)$ and the definition of the index.

Corollary 4.6. If $N_{i} \subset M_{i}$ are inclusions of factors and $\varepsilon_{i} \in C\left(M_{i}, N_{i}\right)$ are faithful expectations $(i=1,2)$ then

$$
\operatorname{Ind}_{\varepsilon_{1} \otimes \varepsilon_{2}}\left(N_{1} \otimes N_{2}, M_{1} \otimes M_{2}\right)=\operatorname{Ind}_{\varepsilon_{1}}\left(N_{1}, M_{1}\right) \operatorname{Ind}_{\varepsilon_{2}}\left(N_{2}, M_{2}\right) .
$$

Proof. If $N_{1}=M_{1}$ or $N_{2}=M_{2}$ the corollary follows immediately by the definition of the index. In general we apply Proposition 4.5 to the inclusions $N_{1} \otimes N_{2} \subset$ $M_{1} \otimes N_{2} \subset M_{1} \otimes M_{2}$ so that

$$
\begin{aligned}
& \operatorname{Ind}_{\varepsilon_{1} \otimes \varepsilon_{2}}\left(N_{1} \otimes N_{2}, M_{1} \otimes M_{2}\right) \\
& \quad=\operatorname{Ind}_{\varepsilon_{1} \otimes \mathrm{id}}\left(N_{1} \otimes N_{2}, M_{1} \otimes N_{2}\right) \operatorname{Ind}_{\mathrm{id} \otimes \varepsilon_{2}}\left(M_{1} \otimes N_{2}, M_{1} \otimes M_{2}\right) \\
& \quad=\operatorname{Ind}_{\varepsilon_{1}}\left(N_{1}, M_{1}\right) \operatorname{Ind}_{\varepsilon_{2}}\left(N_{2}, M_{2}\right) . \quad \bigcirc
\end{aligned}
$$

\section{The Space of the Conditional Expectations}

Extending the analysis made in [22] and in Sect. 3 we study here the relationship between the canonical endomorphism and the conditional expectations and the dependence of the index on the latter.

Let $N \subset M$ be an inclusion of infinite factors and $\gamma=\gamma_{\omega}: M \rightarrow N$ the canonical endomorphism associated with the cyclic state $\omega \in M_{*}$.

The space

$$
H(\gamma)=\{v \in N \mid \gamma(x) v=v x, x \in N\}
$$

is a Hilbert space of isometries in $N$ (possibly with left support less than 1).

In fact if $v, w \in H(\gamma)$ then

thus $w^{*} v \in Z(N)=C$.

$$
w^{*} v x=w^{*} \gamma(x) v=x w^{*} v, \quad x \in N,
$$

Denote by $S$ the unit sphere of $H(\gamma), S=\{v \in H(\gamma) \mid\|v\|=1\}$; every element $v \in S$ is an isometry in $N$.

If we put

$$
\varepsilon_{v}(x)=v^{*} \gamma(x) v, \quad x \in M,
$$

then we get a map $\varepsilon_{v}: M \rightarrow N$; in fact Proposition 5.1 will show that $\varepsilon_{v} \in C(M, N)$ and all normal expectations arise in this way.

Next proposition was obtained in a discussion with J. L. Sauvageot.

Proposition 5.1. The map

$$
v \in S \rightarrow \varepsilon_{v} \in C(M, N)
$$

is a continuous and surjective ( $S$ carries the strong topology and $C(M, N)$ the pointwise weak topology).

Proof. Since $\gamma(M) \subset N$ we have at once that

$$
\varepsilon_{v}(N)=v^{*} \gamma(N) v \subset N .
$$


Moreover if $x \in N$ then

$$
\varepsilon_{v}(x)=v^{*} \gamma(x) v=x v^{*} v=x,
$$

so $\varepsilon_{v} \mid N=$ id. It follows immediately that $\varepsilon_{v}$ is normal and $\varepsilon_{v} \in C(M, N)$.

If $v_{n} \rightarrow v$ strongly, then $v_{n}^{*} \rightarrow v^{*}$ weakly and

$$
\varepsilon_{v_{n}}(x)=v_{n}^{*} \gamma(x) v_{n} \rightarrow v^{*} \gamma(x) v=\varepsilon_{v}(x)
$$

(weak convergence) thus $v \rightarrow \varepsilon_{v}$ is continuous.

It remains to show that surjectivity. Let then $\varepsilon \in C(M, N)$ and put $\varphi \equiv \omega \cdot \varepsilon$, where the cyclic state $\omega=(\cdot \Omega, \Omega)$ is represented by the vector $\Omega$. Let $\xi \in L^{2}(M, \Omega)_{+}$be the vector representative of $\varphi$. The map

$$
V_{0}: x \Omega \rightarrow x \xi, \quad x \in N
$$

determines an isometry $V_{0} \in N^{\prime}$. Set

$$
p=V_{0} V_{0}^{*}=[N \xi] \in N^{\prime},
$$

so that $p=J_{M} p J_{M}$, where $J_{M}$ is the modular conjugation of $L^{2}(M, \Omega)$. If we set

$$
V=J_{N} V_{0} J_{N} \in N,
$$

where $J_{N}$ is the modular conjugation associated with $N, \Omega$ and $\Gamma=J_{N} J_{M}$, then

$$
\Gamma^{*} V=\Gamma^{*} J_{N} V_{0} J_{N}=J_{M} V_{0} J_{N}=J_{M} J_{N}^{\xi} V_{0}=V_{0},
$$

where $J_{N}^{\xi}=\left.J_{M}\right|_{\overline{N \xi}}$ is the modular conjugation of $N p, \xi$ so that $V_{0} J_{N} V_{0}^{*}=J_{N}^{\xi}$.

Hence

$$
V^{*} \gamma(x) V=V^{*} \Gamma x \Gamma^{*} V=V_{0}^{*} x V_{0}=x V_{0}^{*} V_{0}=x, \quad x \in N
$$

and

$$
\begin{aligned}
\varepsilon_{V}(x) & =V^{*} \gamma(x) V=V^{*} \Gamma x \Gamma^{*} V=V_{0}^{*} x V_{0} \\
& =V_{0}^{*} \operatorname{pxp}_{0}=V_{0}^{*} \varepsilon(x) p V_{0}=V_{0} \varepsilon(x) V_{0}^{*}=\varepsilon(x), \quad x \in N .
\end{aligned}
$$

Moreover $V \in H(\gamma)$ because

$$
\gamma(x) V=\Gamma x \Gamma^{*} V=\Gamma x V_{0}=\Gamma V_{0} x=V x, \quad x \in N .
$$

Corollary 5.2. If Ind $(N, M)<\infty$ then $C(M, N)$ is compact.

Proof. By the above theorem it suffices to show that $H(\gamma)$ is finite dimensional. If $\left\{v_{i}, i \in I\right\}$ is an orthonormal basis of $H(\gamma)$, then $e_{i} \equiv v_{i} v_{i}^{*}$ is an orthogonal family of projections in $\gamma(N)^{\prime} \cap N$. However the canonical endomorphism $N \rightarrow \gamma(N)$ equals $\gamma^{2}$ [22] and this implies that Ind $(\gamma(N), N)<\infty$ (taking crossed product) thus $\gamma(N)^{\prime} \cap N$ is finite dimensional and $I$ is a finite set.

Proposition 5.3. Let $N \subset M$ be an inclusion of factors. If there exists a faithful family of conditional expectations in $C(M, N)$, then $C(M, N)$ is affinely homeomorphic to the normal state space $S\left(N^{\prime} \cap M\right)$ of $N^{\prime} \cap M$.

In particular there exists a faithful expectation in $C(M, N)$.

Proof. Let $\gamma: M \rightarrow N$ be the canonical endomorphism and $H(\gamma) \subset N$ be as above. 
Let $z$ be the left support of $H(\gamma)$. Then $z \in \gamma(N)^{\prime} \cap N$ and since

$$
u H(\gamma) u^{*}=H(\gamma)
$$

for all unitaries in $\gamma(N)^{\prime} \cap N, z$ belongs to $Z\left(\gamma(N)^{\prime} \cap M\right)$. By using a maximality argument one obtains $z=1$.

By [22] $\gamma \mid N$ is inner and there exists a faithful conditional expectation in $C(M, N)$.

Now the map

$$
\varepsilon \in C(M, N) \rightarrow \varepsilon \mid N^{\prime} \cap M \in S\left(N^{\prime} \cap M\right)
$$

is affine and continuous. Let $\varphi_{0}$ be a faithful normal state of $N$ and $\varphi=\varphi_{0}{ }^{\circ} \varepsilon$, where $\varepsilon \in C(M, N)$ is faithful. Then $\sigma^{\varphi}$ leaves $N$ and $N^{\prime} \cap M$ stable, hence $\varepsilon$ factors through $N_{1}=N \vee\left(N^{\prime} \cap M\right)$,

$$
\varepsilon=\varepsilon_{2} \cdot \varepsilon_{1}, \quad \varepsilon_{2} \in C\left(N_{1}, N\right), \quad \varepsilon_{1} \in C\left(M, N_{1}\right)
$$

It follows that $N \vee\left(N^{\prime} \cap M\right)$ is naturally isomorphic to $N \otimes\left(N^{\prime} \cap M\right)$ and any state $\varphi \in S\left(N^{\prime} \cap M\right)$ gives a normal conditional expectation $\varepsilon_{\varphi}$ in $C\left(N_{1}, N\right)$.

Note that we have $N_{1}^{\prime} \cap M \subset N_{1}$, hence $C\left(M, N_{1}\right)$ contains only one element $\varepsilon_{1}$. The conditional expectation $\varepsilon_{\varphi} \cdot \varepsilon_{1}$ obviously satisfies

$$
\varepsilon_{\varphi} \cdot \varepsilon_{1} \mid N^{\prime} \cap M=\varphi
$$

and we have a continuous inverse of $\varepsilon \rightarrow \varepsilon \mid N^{\prime} \cap M$.

It is now not difficult to check that the map

$$
\varepsilon \in C(M, N) \rightarrow \operatorname{Ind}_{\varepsilon}(N, M) \in[1,+\infty]
$$

is continuous and has a convex restriction to expectations corresponding to tracial states of $N^{\prime} \cap M$. If Ind $(N, M)<\infty$, it attains a minimum at a unique point.

We shall in fact give an explicit formula for such a minimal conditional expectation based on a formula in [18] (next Proposition 5.4), that we prove in our setting.

If $N \subset M$ are factors and $e$ is a projection of $N^{\prime} \cap M$, then for any faithful $\varepsilon \in C(M, N)$ the formula

$$
\varepsilon_{p}(x)=\varepsilon(p)^{-1} \varepsilon(x) p \quad x \in M_{p}
$$

defines an element of $C\left(M_{p}, N_{p}\right)$.

Proposition 5.4. If $\left\{p_{i}\right\}$ is a partition of the unity in $N^{\prime} \cap M$ that belongs to the centralizer of $\varepsilon \mid N^{\prime} \cap M$ then

$$
\operatorname{Ind}_{\varepsilon}(N, M)=\sum_{i} \varepsilon\left(p_{i}\right)^{-1} \operatorname{Ind}_{\varepsilon_{p_{i}}}\left(N_{p_{i}}, M_{p_{i}}\right) .
$$

Proof. With the notations of Sect. $4, p_{i}$ belongs to $Z\left(\tilde{N}^{\prime} \cap \tilde{M}\right)$. The problem is thus reduced to the semifinite case, (inclusions of semifinite von Neumann algebras with a centrally ergodic trace) hence to the finite case by Proposition 1.3 and one applies the argument in [19, Lemma 2.2.2] by using Lemma 3.2.

Notice that if $\operatorname{Ind}(N, M)<\infty$ then $N^{\prime} \cap M$ is finite dimensional; if $N^{\prime} \cap M=\mathrm{C}$ 
then $C(M, N)$ contains only one element; if $N^{\prime} \cap M$ is a factor there exists a canonical expectation $\varepsilon \in C(M, N)$, namely the one such that $\varepsilon \mid N^{\prime} \cap M$ is the trace of $N^{\prime} \cap M$. It follows straightforwardly by Proposition 4.5 that this canonical expectation is the unique one that minimizes Ind $(M, N)$.

If $N^{\prime} \cap M$ is not a factor we choose a partition of the unit by minimal projections $z_{i} \in Z\left(N^{\prime} \cap M\right)$. Proposition 5.4 shows that the minimum of $\operatorname{Ind}_{\varepsilon}(M, N)$ is taken when $\varepsilon \mid N^{\prime} \cap M$ is a trace and to specify $\varepsilon$ we should determine the coefficients $\varepsilon\left(z_{i}\right)$.

Theorem 5.5. If $N \subset M$ is an inclusion of factors with finite index, there exists a unique conditional expectation $\varepsilon \in C(M, N)$ that minimizes $\operatorname{Ind}_{\varepsilon}(N, M)$.

If $\left\{z_{i}\right\}$ is the family of the minimal central projection of $N^{\prime} \cap M$, then $\varepsilon$ is given by

$$
\begin{aligned}
& \varepsilon \mid N^{\prime} \cap M \text { is a trace } \\
& \varepsilon\left(z_{i}\right)=\frac{I_{i}^{1 / 2}}{\sum_{i} I_{i}^{1 / 2}},
\end{aligned}
$$

where $I_{i} \equiv \operatorname{Ind}\left(N z_{i}, M z_{i}\right)$ is the index of $N z_{i}$ of $M z_{i}$ given by the trace of $\left(N^{\prime} \cap M\right) z_{i}$.

The minimal index is given by

$$
\operatorname{Ind}(N, M)^{1 / 2}=\sum_{i} I_{i}^{1 / 2} .
$$

More generally the above formula holds if $\left\{z_{i}\right\}$ is any partition of the unity by projections in $\varepsilon \mid N^{\prime} \cap M$ and $I_{i}$ denotes the minimal index $\operatorname{Ind}\left(N z_{i}, M z_{i}\right)$.

Proof. By the above considerations and Proposition 5.4 we must minimize the expression

$$
\sum_{i} t_{i}^{-1} I_{i}
$$

with the conditions $t_{i}>0, \sum t_{i}=1$. The theorem is then obtained by an elementary application of the Lagrange multipliers theorem.

Remark. If $N \subset M$ is an inclusion of finite factors with finite index, the minimal expectation in $C(M, N)$ does not always coincide with the trace preserving conditional expectation. This fact is related with the inequality between the entropy and the logarithm of the index [29].

Corollary 5.6. If $N_{i} \subset M_{i}$ are inclusions of factors $(i=1,2)$,

$$
\operatorname{Ind}\left(N_{1} \otimes N_{2}, M_{1} \otimes M_{2}\right)=\operatorname{Ind}\left(N_{1}, M_{1}\right) \operatorname{Ind}\left(N_{2}, M_{2}\right) \text {. }
$$

Proof. It is easily seen that we may assume that $\operatorname{Ind}\left(N_{i}, M_{i}\right)$ is finite $(i=1,2)$. Let then $\varepsilon_{i} \in C\left(M_{i}, N_{i}\right)$ be the minimal expectations. By Corollary 4.6 we have only to show that $\varepsilon_{1} \otimes \varepsilon_{2}$ is the minimal expectation in $C\left(M_{1} \otimes M_{2}, N_{1} \otimes N_{2}\right)$. Since the relative commutant of $N_{1} \otimes N_{2}$ in $M_{1} \otimes M_{2}$ coincides with $\left(N_{1}^{\prime} \cap M_{1}\right) \otimes\left(N_{2} \cap M_{2}\right)$, Theorem 5.5 immediately entails the corollary.

For future use we include the following.

Proposition 5.7. If $N \subset M$ is a finite index inclusion of factors, then every conditional expectation $\varepsilon$ of $M$ onto $N$ is normal. 
Proof. By a straightforward extension of the argument in [29, Proposition 1.3] to arbitrary factors, $M$ is a finitely generated right $N$-module, i.e. there exist finitely many elements $\lambda_{1}, \ldots, \lambda_{n} \in M$ such that every $x \in M$ may be written uniquely as

$$
x=\sum x_{i} \lambda_{i}, \quad x_{i} \in N .
$$

It follows that

thus $\varepsilon$ is normal.

$$
\varepsilon(x)=\sum x_{i} \varepsilon\left(\lambda_{i}\right)
$$

\section{Index and Compact Actions}

Let $M$ be a von Neumann algebra and $\alpha: G \rightarrow$ Aut $(M)$ an action of a compact group $G$ on $M$. We denote by $E$ the $\alpha$-invariant conditional expectation of $M$ onto the fixed point algebra $M^{\alpha}$,

$$
E \equiv \int \alpha_{g}(\cdot) d g .
$$

If $\varphi_{0} \in M_{*}^{\alpha}$ is a faithful state of $M^{\alpha}$ then $\varphi \equiv \varphi_{0} \cdot E \in M_{*}$ is a faithful $\alpha$-invariant state of $M$. The modular group $\sigma^{\varphi}$ of $M$ associated with $\varphi$ restricts to $\sigma^{\varphi_{0}}$ on $M^{\alpha}$ and we have the inclusions

$$
\begin{array}{ccc}
M^{\alpha} & \subset & M \\
\cap & & \cap \\
M^{\alpha} \times{ }_{\sigma^{\varphi_{0}}} \mathbf{R} & \subset M \times_{\sigma^{\varphi}} \mathbf{R}
\end{array}
$$

Lemma 6.1. The dual trace $\tau$ of $M \times{ }_{\sigma^{\phi}} \mathbf{R}$ restricts to the dual trace $\tau_{0}$ of $M^{\alpha} \times{ }_{\sigma^{\varphi_{0}}} \mathbf{R}$.

Proof. The argument is the same as the one given in Sect. 4. By using the notations there given, $\tau=\tilde{\varphi}(H \cdot)$ and $\tau_{0}=\varphi_{0}(H \cdot)$, where $U_{t} \equiv H^{i t}$ are the usual unitaries in $M^{\alpha} \times{ }_{\sigma^{\varphi_{0}}} \mathbf{R} \subset M \times{ }_{\sigma} \mathbf{R}$. Since $\tilde{\varphi}$ extends $\tilde{\varphi}_{0}$ also $\tau$ extends $\tau_{0}$.

Note now that the action $\alpha$ extends to an action $\tilde{\alpha}: G \rightarrow \operatorname{Aut}(\tilde{M})$, where $\tilde{M} \equiv M \times{ }_{\sigma} \mathbf{R}$, determined by

$$
\tilde{\alpha}_{g} \mid M=\alpha_{g}, \quad \tilde{\alpha}_{g}\left(U_{t}\right)=U_{t}, \quad t \in \mathbf{R} \quad g \in G,
$$

where the $U_{t}$ are the usual unitaries in $\tilde{M}$, as follows easily because $\alpha$ and $\sigma^{\varphi}$ commute.

Lemma 6.2. The $\tilde{\alpha}$-fixed point subalgebra $\tilde{M}^{\tilde{\alpha}}$ of $\tilde{M}$ is $M^{\alpha} \times{ }_{\sigma^{\varphi_{0}}} \mathbf{R}$.

Proof. Obviously $M^{\alpha} \times{ }_{\sigma^{\varphi_{0}}} \mathbf{R}$ is contained in $\tilde{M}^{\tilde{\alpha}}$. To show the reverse inclusion we consider the conditional expectation $\widetilde{E} \in C\left(\tilde{M}, \tilde{M}^{\tilde{\alpha}}\right)$,

$$
\tilde{E} \equiv \int \tilde{\alpha}_{g} d g .
$$

By the definition of $\tilde{\alpha}$, we have

$$
\widetilde{E} \mid M=E, \quad \widetilde{E}\left(U_{t}\right)=U_{t},
$$

in particular $\tilde{E}(M)=M^{\alpha}$. Since the finite sums

$$
x=\sum x_{i} U_{t_{i}} \quad x_{i} \in M, \quad t_{i} \in \mathbf{R},
$$


form a weakly dense subalgebra of $\tilde{M}$ and $\tilde{E}$ is normal, from

$$
\tilde{E}(x)=\sum E\left(x_{i}\right) U_{t_{i}} \in M^{\alpha} \times{ }_{\sigma_{0}{ }^{\varphi} \mathbf{R}},
$$

we deduce that $\tilde{M}^{\tilde{\alpha}} \subset M^{\alpha} \times{ }_{\sigma^{\phi_{0}}} \mathbf{R}$.

For simplicity we assume now that $M$ is a factor and consider a $\alpha$-invariant subfactor $N$ of $M$. Suppose $\varepsilon \in C(M, N)$ is a faithful conditional expectation that commutes with $\alpha$, so that $\varepsilon_{0} \equiv \varepsilon \mid M^{\alpha} \in C\left(M^{\alpha}, N^{\alpha}\right)$.

Theorem 6.3. If $\alpha \mid N$ is dominant then $\operatorname{Ind}_{\varepsilon}(N, M)=\operatorname{Ind}_{\varepsilon_{0}}\left(N^{\alpha}, M^{\alpha}\right)$.

Before proving the theorem recall that an action $\alpha: G \rightarrow \operatorname{Aut}(N)$ of a compact group $G$ on a von Neumann algebra $N$ is dominant if $N^{\alpha}$ is properly infinite and the monodial spectrum of $\alpha$ is complete, i.e. for every $\pi \in \widehat{G}$ there is a $\alpha$-invariant Hilbert space of isometries $H$ in $N$ such that $\alpha \mid H$ is equivalent to $\pi$, see [30,24].

Elementary examples (e.g. with $M$ a finite dimensional factor and $N=C$ ) show that Theorem 6.3 may fail without the dominance assumption, instead one has the following.

Proposition 6.4. With the above notations

$$
\operatorname{Ind}_{\varepsilon}(N, M) \geqq \operatorname{Ind}_{\varepsilon_{0}}\left(N^{\alpha}, M^{\alpha}\right)
$$

(without any dominance assumption).

Proof. If $\lambda=\operatorname{Ind}_{\varepsilon}(N, M)^{-1}$, then by Theorem 4.1,

$$
\varepsilon(x) \geqq \lambda x \quad x \in M^{+},
$$

hence

$$
\varepsilon_{0}(y)=\varepsilon(y) \geqq \lambda y, \quad y \in M^{\alpha^{+}},
$$

showing the desired inequality again by Theorem 4.1 .

Lemma 6.5. Let $\alpha: G \rightarrow \operatorname{Aut}(M, N)$ be an action of a compact group $G$ on the inclusion of von Neumann algebras $N \subset M$. If $\omega \in M_{*}$ is a $\alpha$-invariant cyclic state for $N \subset M$, then $\omega_{0} \equiv \omega \mid M^{\alpha}$ is a cyclic state for $N^{\alpha} \subset M^{\alpha}$ and the canonical endomorphism $\gamma_{\omega_{0}}: M^{\alpha} \rightarrow N^{\alpha}$ is the restriction to $M^{\alpha}$ of $\gamma_{\omega}: M \rightarrow N$.

Proof. Let $\Omega$ be the vector representative for $\omega$, cyclic and separating for $N$ and $M$, and $U$ the $\Omega$-fixing unitary implementation of $\alpha$, i.e. $U_{g} x \Omega=\alpha_{g}(x) \Omega, x \in M, g \in G$. The subspaces $\left[M^{\alpha} \Omega\right]$ and $\left[N^{\alpha} \Omega\right]$ coincide since they are both equal to the subspace of the $U$-fixed vectors, therefore $\omega_{0}$ is a cyclic state for $N^{\alpha} \subset M^{\alpha}$. Since $\left[M^{\alpha} \Omega\right]$ is an invariant subspace for the modular conjugations of $M$ and $N$ with respect to $\Omega$ [32], it follows immediately that $\gamma_{\omega}$ restricts to $\gamma_{\omega_{0}}$.

Proof of Theorem 6.3. Let $\varphi_{0}$ be a faithful normal state of $N^{\alpha}$ and

$$
\varphi \equiv \varphi_{0} \cdot \varepsilon \cdot E \in M_{*}
$$

the extension of $\varphi_{0}$ to $M$ by $\varepsilon \cdot E$ (since $E$ and $\varepsilon$ commute $\varepsilon \cdot E \in C\left(M, N^{\alpha}\right)$ ). The modular group $\sigma^{\varphi}$ of $\varphi$ leaves $N, M^{\alpha}$ and $N^{\alpha}$ globally invariant; if we also denote by $\sigma^{\varphi}$ restrictions of $\sigma^{\varphi}$, we have to show by Lemmas 6.1 and 6.2 that the index 
of $N \times{ }_{\sigma^{\varphi}} \mathbf{R}$ in $M \times{ }_{\sigma^{\varphi}} \mathbf{R}$ with respect to the dual trace $\tau$ of $M \times{ }_{\sigma^{\varphi}} \mathbf{R}$ is equal to the index of $N^{\alpha} \times{ }_{\sigma^{\varphi}} \mathbf{R}$ in $M^{\alpha} \times{ }_{\sigma^{\varphi}} \mathbf{R}$ with respect to $\tau_{0} \equiv \tau \mid M^{\alpha}$ :

$$
\operatorname{Ind}_{\tau_{0}}\left(\tilde{N}^{\tilde{\alpha}}, \tilde{M}^{\tilde{\alpha}}\right)=\operatorname{Ind}_{\tau}(\tilde{N}, \tilde{M}) \text {. }
$$

Let $\tilde{E}=\int \tilde{\alpha}_{g} d g \in C(\tilde{M}, \tilde{N})$ be as in Lemma 6.2. Since $\alpha \mid N$ is dominant and $\tilde{\alpha}|N=\alpha| N$ also $\tilde{\alpha} \mid \tilde{N}$ is dominant.

Let $\omega_{0} \in \tilde{M}_{*}$ be a cyclic state for $\tilde{N} \subset \tilde{M}$ (we may assume that $N$ and $M$ are infinite) and

$$
\omega=\omega_{0} \cdot \tilde{E} \in \tilde{M}_{*}
$$

As in [24] $\omega$ is a cyclic state for $\tilde{N} \subset \tilde{M}$ and by Lemma 6.4 the canonical endomorphism $\gamma_{\omega_{0}}: \tilde{M}^{\tilde{\alpha}} \rightarrow \tilde{N}^{\tilde{\alpha}}$ is the restriction of $\gamma_{\omega}: \tilde{M} \rightarrow \tilde{N}$; since

$$
\begin{aligned}
& \tau\left(\gamma_{\omega}(x)\right)=\tau(h x), \quad x \in \tilde{M}, \\
& \tau\left(\gamma_{\omega}(x)\right)=\tau_{0}\left(\gamma_{\omega_{0}}(x)\right)=\tau_{0}(k x)=\tau(k x), \quad x \in \tilde{M}^{\alpha},
\end{aligned}
$$

where $h=\bmod _{\tau}\left(\gamma_{\omega}\right), k=\bmod _{\tau_{0}}\left(\gamma_{\omega_{0}}\right)$, it follows that $\tilde{E}(h)=k$. Since $M$ is a factor $h \in[1, \infty]$ and $\tau_{0}$ is a scalar trace

$$
\operatorname{Ind}_{\tau_{0}}\left(\tilde{N}^{\tilde{\alpha}}, \tilde{M}^{\tilde{\alpha}}\right)=\operatorname{Ind}_{\tau}(\tilde{N}, \tilde{M}) .
$$

Denoting as before by Ind $(N, M)$ the minimal index of $N$ in $M$, a natural question is whether $\operatorname{Ind}\left(N^{\alpha}, M^{\alpha}\right)=\operatorname{Ind}(N, M)$ in the above theorem, namely whether the minimal conditional expectation $\varepsilon \in C(M, N)$ restricts to the minimal conditional expectation $\varepsilon_{0} \in C\left(M^{\alpha}, N^{\alpha}\right)$. We give here a positive answer under conditions that suffice for our applications.

Recall that the centralizer $M^{\varepsilon}$ of $\varepsilon$ on $M$ is equal to $\{x \in M \mid \varepsilon(x y)=\varepsilon(y x), y \in M\}$.

Corollary 6.6. Let $\alpha: G \rightarrow \operatorname{Aut}(M, N)$ be as in Theorem 6.3 with $N^{\alpha}, M^{\alpha}$ factors. Assume further that there exists a partition of the unit by projections $e_{i}$ of $\left(N^{\prime} \cap M\right)^{\alpha}$ such that

$$
e_{i} \in M^{\varepsilon}, \quad\left(N^{\alpha^{\prime}} \cap M^{\alpha}\right) e_{i}=\mathbf{C} e_{i} .
$$

Then $\operatorname{Ind}(N, M)=\operatorname{Ind}\left(N^{\alpha}, M^{\alpha}\right)$.

Proof. First we assume that $\left\{e_{i}\right\}$ contains only one element, i.e. $N^{\alpha^{\prime}} \cap M^{\alpha}=$ C. In this case there exists a unique element $\varepsilon_{0}$ in $C\left(M^{\alpha}, N^{\alpha}\right)$; if $\varepsilon \in C(M, N)$ is the minimal expectation (we assume $N \subset M$ has finite index) then $\varepsilon$ commutes with $\alpha$ by canonicity and $\varepsilon \mid M^{\alpha}=\varepsilon_{0}$ and Theorem 6.3 applies. In general we denote by

$$
I_{i}=\operatorname{Ind}\left(N_{e_{i}}, M_{e_{i}}\right)
$$

the minimal index, then by the above comments

$$
I_{i}=\operatorname{Ind}\left(N_{e_{i}}, M_{e_{i}}\right)=\operatorname{Ind}\left(N_{e_{i}}^{\alpha}, M_{e_{i}}^{\alpha}\right) .
$$

Since $e_{i} \in M^{\varepsilon}$ also $e_{i} \in\left(M^{\alpha}\right)^{\varepsilon_{0}}$; by Theorem 5.5,

$$
\operatorname{Ind}\left(N^{\alpha}, M^{\alpha}\right)^{1 / 2}=\sum I_{i}^{1 / 2}=\operatorname{Ind}(N, M)^{1 / 2}
$$

that concludes the proof. 
Corollary 6.7. With the assumptions of Theorem 6.3, if $G$ is abelian then

$$
\operatorname{Ind}\left(N^{\alpha}, M^{\alpha}\right)=\operatorname{Ind}(N, M)
$$

assuming that $N^{\alpha}, M^{\alpha}$ are factors.

Proof. We may assume Ind $(N, M)<\infty$. Let $\varepsilon \in C(M, N)$ the minimal expectation, we have to show that $\varepsilon_{0} \equiv \varepsilon \mid M^{\alpha}$ be the minimal expectation $\varepsilon_{0}^{\prime}$ in $C\left(M^{\alpha}, N^{\alpha}\right)$. It is sufficient to show that $\varepsilon_{0}^{\prime}$ extends to a faithful $\alpha$-invariant expectation $\varepsilon^{\prime}$ in $C(M, N)$, since then we have by Theorem 6.3

$$
\operatorname{Ind}_{\varepsilon^{\prime}}(M, N)=\operatorname{Ind}_{\varepsilon_{0}^{\prime}}\left(M^{\alpha}, N^{\alpha}\right) \leqq \operatorname{Ind}_{\varepsilon_{0}}\left(M^{\alpha}, N^{\alpha}\right)=\operatorname{Ind}_{\varepsilon}(M, N)=\operatorname{Ind}(M, N),
$$

and $\varepsilon=\varepsilon^{\prime}$ by the uniqueness of $\varepsilon$ given in Theorem 5.5 .

To this end let $\omega_{0} \in M_{*}^{\alpha}$ be a faithful $\varepsilon_{0}^{\prime}$-invariant state, $\omega=\omega_{0} \cdot E$ and $\theta: \hat{G} \rightarrow \operatorname{Aut}\left(M^{\alpha}, N^{\alpha}\right)$ the dual action of $\alpha$. By its uniqueness $\varepsilon_{0}^{\prime}$ commutes with $\theta$, thus the modular group $\sigma^{\omega}$ of $\omega$ leaves $N$ globally invariant [32] and we have a conditional expectation $\varepsilon^{\prime} \in C(M, N)$ that leaves $\omega$ fixed. Since $\varepsilon^{\prime} \mid M^{\alpha}$ leaves $\omega_{0}$ fixed $\varepsilon^{\prime} \mid M^{\alpha}=\varepsilon_{0}^{\prime}$ as desired.

The implications of Theorem 6.3 are also examplified by the Galois correspondence established in [1] that we state in the case of a prime compact action.

Corollary 6.8 [1]. Let $\alpha: G \rightarrow \operatorname{Aut}(M)$ be an action of a compact group $G$ on a factor $M$ such that $M^{\alpha^{\prime}} \cap M=\mathbf{C}$. If $N \subset M$ is a $\alpha$-invariant von Neumann subalgebra that contains $M^{\alpha}$, there exists a closed normal subgroup $H$ of $G$ such that $N=\left\{x \in M \mid \alpha_{g}(x)=x, g \in H\right\}$.

Proof. By considering a quotient of $G$ we may assume that $g \rightarrow \alpha_{g} \mid N$ is one-to-one and show that $N=M$. By tensoring $M$ by a type $I_{\infty}$ factor we may assume that $M^{\alpha}$ is infinite, thus $\alpha \mid N$ is dominant. By Theorem 6.3 we have

$$
\operatorname{Ind}_{\varepsilon}(N, M)=\operatorname{Ind}\left(M^{\alpha}, N^{\alpha}\right)=1,
$$

thus $N=M$ (the conditional expectation $\varepsilon \in C(M, N)$ exists by an argument in [27]).

The interest in the above proof stays in the fact that extends to the case of locally compact actions [27].

We now restrict our attention to a special case of Corollary 6.6 that will appear in the next section.

Let $\alpha: G \rightarrow \operatorname{Aut}(M)$ be an action of a compact group $G$ on the factor $M$ and denote by $\mathscr{H}^{\alpha}(M)$ the set of the $\alpha$-invariant Hilbert spaces in $M$, see $[30,24]$; if $H \in \mathscr{H}^{\alpha}(M)$, the inner endomorphism $\varrho_{H}$ of $M$ implemented by $H$

$$
\varrho_{H}(x)=\sum_{i} v_{i} x v_{i}^{*}, \quad x \in M,
$$

where $\left\{v_{i}, i \in I\right\}$ is a basis for $H$, depends only on $H$ and not on the basis, hence $\varrho_{H}$ commutes with $\alpha$. In particular $\varrho_{H}$ restricts to an endomorphism $\varrho_{0}$ of the fixed point algebra $M^{\alpha}$.

Proposition 6.9. If $M^{\alpha^{\prime}} \cap M=\mathbf{C}$ and $\alpha \mid H$ is irreducible, then $\varrho_{0}\left(M^{\alpha}\right)^{\prime} \cap M^{\alpha}=\mathbf{C}$. 
Proof. We only sketch the proof of this proposition. The detailed proof will be found in [27].

Let $x \in \varrho_{0}\left(M^{\alpha}\right)^{\prime} \cap M^{\alpha}$, namely $x \in M^{\alpha}$ and

$$
\varrho_{0}(y) x=x \varrho_{0}(y), \quad y \in M^{\alpha}
$$

or

$$
\sum_{i} v_{i} y v_{i}^{*} x=\sum_{i} x v_{i} y v_{i}^{*} \quad y \in M^{\alpha}
$$

with $\left\{v_{i}, i \in I\right\}$ a basis of $H$ as above. Multiplying both sides of this equality by $v_{h}^{*}$ from the left and by $v_{k}$ from the right we get

$$
y v_{h}^{*} x v_{k}=v_{h}^{*} x v_{k} y, \quad y \in M^{\alpha},
$$

namely $v_{h}^{*} x v_{k} \in M^{\alpha^{\prime}} \cap M=\mathbf{C}$.

Let $\Psi$ be the isomorphism of $M$ onto $\operatorname{Mat}_{n}(M)=M \otimes F_{n}$, where $F_{n}$ is the type $I_{n}$ factor, $n=\operatorname{dim}(H)$ given by

$$
\Psi(a)=\left\{v_{h}^{*} a v_{k}\right\}, \quad h, k \in I,
$$

then $\Psi$ intertwines $\alpha$ with $\alpha \otimes \operatorname{ad} \pi$, where $\pi \in \widehat{G}$ is given by $\alpha \mid H$.

The condition $v_{h}^{*} x v_{k} \in \mathbf{C}$ then means

$$
\Psi(x) \in 1 \otimes F_{n} .
$$

Since $x \in M^{\alpha}$, we have $\Psi(x) \in\left(M \otimes F_{n}\right)^{\alpha \otimes \text { ad } \pi}$ thus $\Psi(x) \in \mathbf{C}$ because $\pi$ is irreducible.

Let $H$ be as above. Setting

$$
(H, H)=\text { lin. } \operatorname{span}\left\{v w^{*} \mid v, w \in H\right\},
$$

recall that $(H, H)$ is a type $I$ factor isomorphic to $B(H)$ and one has the tensor product decomposition [30]

$$
M \simeq \varrho_{H}(M) \otimes(H, H) .
$$

Corollary 6.10. With the above notations assume that $M^{\alpha^{\prime}} \cap M=\mathbf{C}$, then

$$
\text { Ind }\left(\varrho_{0}\left(M^{\alpha}\right), M^{\alpha}\right)=(\operatorname{dim} H)^{2} .
$$

Proof. We may assume that $M$ is infinite. We notice first that $\alpha \mid \varrho_{H}(M)$ is dominant, in fact $\alpha$ commutes with $\varrho_{H}$ thus $\alpha \mid \varrho_{H}(M)=\varrho_{H} \cdot \alpha \cdot \varrho_{H}^{-1}$ and $\alpha$ is dominant. By Theorem 6.3,

$$
\operatorname{Ind}_{\varepsilon_{0}}\left(\varrho_{0}\left(M^{\alpha}\right), M^{\alpha}\right)=(\operatorname{dim} H)^{2},
$$

where $\varepsilon_{0} \in C\left(M^{\alpha}, \varrho_{0}\left(M^{\alpha}\right)\right)$ is the restriction to $M^{\alpha}$ of the expectation $\varepsilon \in C(M, \varrho(M))$ given by the trace on $\varrho(M)^{\prime} \cap M=(H, H)$, i.e. $\varepsilon$ is the minimal conditional expectation. In fact by the example following Proposition 1.3 and by Corollary 5.6 we have

$$
\text { Ind }\left(\varrho_{H}(M), M\right)=(\operatorname{dim} H)^{2} .
$$

We have to show that $\varepsilon_{0}$ is the minimal conditional expectation in $C\left(M^{\alpha}, \varrho_{0}\left(M^{\alpha}\right)\right)$. 
If $\alpha \mid H$ is irreducible it follows by Proposition 6.9 that $\varrho_{0}\left(M^{\alpha}\right)^{\prime} \cap M^{\alpha}=\mathbf{C}$, thus $C\left(M^{\alpha}, \varrho_{0}\left(M^{\alpha}\right)\right)$ contains only one element and we have nothing to prove.

In general we consider the partition of the unity by the projections $e_{j} \in(H, H)=$ $\varrho(M)^{\prime} \cap M$ onto the irreducible components of $\alpha \mid H$. Then the $e_{j}$ are $\alpha$-invariant and $\left(\varrho\left(M^{\alpha}\right)^{\prime} \cap M^{\alpha}\right) e_{j}=\mathbf{C} e_{j}$ because of Proposition 6.9, and we may apply Corollary 6.6 to conclude.

By the above corollary we have in particular examples of subfactors with trivial relative commutant and index $n^{2}, n \in \mathbf{N}$, that in general do not come from the crossed product construction.

Theorem 6.11. Let $M$ be a factor (finite or infinite) and $\alpha: G \rightarrow \operatorname{Aut}(M)$ an action of a compact group with $M^{\alpha^{\prime}} \cap M=\mathbf{C}$. If $\pi$ is a unitary representation of $G$ on a Hilbert space $H$ of dimension $n$, then

$$
\text { Ind }\left(M^{\alpha} \otimes 1,(M \otimes B(H))^{\alpha \otimes a d \pi}\right)=n^{2}
$$

and if $\pi$ is irreducible $M^{\alpha} \otimes 1$ has trivial relative commutant in $(M \otimes B(H))^{\alpha \otimes \mathrm{ad} \pi}$.

Proof. By tensoring $M$ with a type $I_{\infty}$ factor we may assume that $M$ is infinite, thus $\alpha$ is dominant. Let $H \in \mathscr{H}^{\alpha}(M)$ be chosen such that $\alpha \mid H$ is equivalent to $\pi$. Then we have a tensor product decomposition $M \simeq \varrho_{H}(M) \otimes(H, H)$, where $\alpha$ becomes $\alpha \otimes$ ad $\pi$ (see the proof of Proposition 6.9). The rest follows by Corollary 6.10 .

It would be interesting to extend Theorem 6.11 to quantum groups and square integrable representations of locally compact groups.

\section{Index and Parastatistics}

In this section we shall exhibit a connection between the statistical dimension of a superselection sector in Quantum Field Theory and the index of certain subfactors, that will rely on the previous analysis in this paper (local observable von Neumann algebras are properly infinite, see [25]).

Let

$$
\mathcal{O} \in \mathbf{R}^{d} \rightarrow \mathscr{A}(\mathcal{O})
$$

be the net of local observable von Neumann algebras associated with the set $\mathscr{K}$ of double cones of the Minkowski space of space time dimension $d$.

In this section we assume $d>2$ so that the analysis is [7] is applicable. There is an analogous version of our results in the case of charges localized in spacelike cones [4], provided $d>3$.

We use the usual assumptions on the net $\mathscr{A}(\mathcal{O})$ as in [7]. In particular we assume Haag duality

$$
\mathscr{A}(\mathcal{O})=\mathscr{A}\left(\mathcal{O}^{\prime}\right)^{\prime}, \quad \mathcal{O} \in \mathscr{K},
$$

where $\mathcal{O}^{\prime}$ denotes the space-like complement of $\mathcal{O}$, and for an unbounded region $\mathcal{O} \in \mathbf{R}^{d}, \mathscr{A}(\mathcal{O})$ is defined as the $C^{*}$-algebra generated by $\left\{\mathscr{A}\left(\mathcal{O}_{1}\right) ; \mathcal{O}_{1} \subset \mathcal{O}, \mathcal{O}_{1} \in \mathscr{K}\right\}$.

In a Wightman theory duality automatically holds for the net $\mathcal{O} \rightarrow \mathscr{A}\left(\mathcal{O}^{\prime}\right)^{\prime}$ that may be used as well in our analysis [2].

As explained in [7] the physically relevant representations of the quasi-local 
$C^{*}$-algebra

$$
\mathscr{A}=\bigcup \mathscr{A}(\mathcal{O})^{-}
$$

(norm closure, union over $\mathcal{O} \in \mathscr{K}$ ) are realized as localized endomorphisms of $\mathscr{A}$, namely endomorphisms $\varrho$ of $\mathscr{A}$ that restrict to the identity on $\mathscr{A}\left(\mathcal{O}^{\prime}\right)$ for some $\mathscr{O} \in \mathscr{K}(\varrho$ is localized in $\mathcal{O})$ at least in short range interaction theories.

Denote by $\mathscr{E}$ the semigroup of all localized endomorphisms of $\mathscr{A}$. Note that if $\varrho \in \mathscr{E}$ is localized in $\mathcal{O}$, then if $T \in \mathscr{A}(\mathcal{O}), S \in \mathscr{A}\left(\mathcal{O}^{\prime}\right)$,

$$
\varrho(T) S=\varrho(T S)=\varrho(S T)=S \varrho(T),
$$

thus by duality

$$
\varrho(\mathscr{A}(\mathcal{O})) \subset \mathscr{A}\left(\mathcal{O}^{\prime}\right)^{\prime}=\mathscr{A}(\mathcal{O}),
$$

and we get inclusions of von Neumann algebras $\varrho(\mathscr{A}(\mathcal{O})) \subset \mathscr{A}(\mathcal{O})(\varrho$ is automatically locally normal).

Two endomorphisms $\varrho, \varrho^{\prime} \in \mathscr{E}$ are equivalent if they are equivalent as representations of $\mathscr{A}$; equivalently there exists a unitary $u \in \mathscr{A}$ such that

$$
\varrho^{\prime}(T)=u \varrho(T) u^{*}, \quad T \in \mathscr{A}
$$

(automatically $u \in \mathscr{A}(\mathcal{O})$ for some $\mathcal{O} \in \mathscr{K}$ ).

Let $\mathscr{E}_{c}$ be the semigroup of the $\varrho \in \mathscr{E}$ that are equivalent to all their space-time translated. The set of the equivalence classes of $\mathscr{E}_{c}$ corresponds to the superselection sectors.

If $\varrho \in \mathscr{E}_{c}$ one chooses $\varrho^{\prime} \in \mathscr{E}_{c}$ in the same equivalence class $[\varrho]$ of $\varrho$ so that $\varrho$ and $\varrho^{\prime}$ are localized in space-like separated regions and a unitary $u \in \mathscr{A}$ such that (7.1) holds. The unitary

$$
\epsilon_{\varrho} \equiv u^{*} \varrho(u)
$$

depends only on $\varrho$ and not on $\varrho^{\prime}, u$; moreover $\varepsilon$ is selfadjoint (one uses here the assumption $d>2$ ).

Associated with $\varrho$ there exists a left inverse $\phi$ of $\varrho$, i.e. a completely positive $\operatorname{map} \phi: \mathscr{A} \rightarrow \mathscr{A}$ with

$$
\phi \cdot \varrho=\mathrm{id} .
$$

If $\varrho$ is irreducible the statistic parameter of $\varrho$

$$
\lambda_{\varrho}=\phi\left(\epsilon_{\varrho}\right)
$$

is a scalar depending only on [@]. Its possible values are

$$
\lambda_{\varrho}=0, \pm 1, \pm \frac{1}{2}, \pm \frac{1}{3}, \ldots
$$

More generally if $\varrho$ is reducible there exists a standard left inverse $\phi$, namely a left inverse such that $\lambda_{\varrho}^{2}=\phi\left(\varepsilon_{\varrho}\right)^{2}$ is a scalar. The inverse of $\left|\lambda_{\varrho}\right|$

$$
d(\varrho)=\left|\lambda_{\varrho}\right|^{-1}
$$

(with the convention $\left.0^{-1}=\infty\right)$ is the statistical dimension of $\varrho$. If $d(\varrho)<\infty(\varrho$ has finite statistics) the standard left inverse is unique. 
For simplicity we shall assume in the sequel that $\mathscr{A}(\mathcal{O}), \mathcal{O} \in \mathscr{K}$, is a factor. This assumption, rather general, can be avoided either with minor modifications of the arguments or replacing $\mathcal{O}$ with a wedge region $W$ (a Poincare translated of the region $\left.x_{1}>\left|x_{0}\right|\right)$, since it follows automatically that $\mathscr{A}(W)^{\prime \prime}$ is a factor of type $I I I_{1}$, see $[25]$.

Theorem 7.1. If $\varrho \in \mathscr{E}_{c}$ is an endomorphism of $\mathscr{A}$ localized in the double cone $\mathscr{O}_{\varrho} \in \mathscr{K}$, then

$$
d(\varrho)^{2}=\operatorname{Ind}[\varrho(\mathscr{A}(\mathcal{O})), \mathscr{A}(\mathcal{O})]
$$

for all $\mathcal{O} \in \mathscr{K}$ that contains $\mathcal{O}_{\rho}$.

Notice that Theorem 7.1 states that $d(\varrho)^{2}$ is equal to the minimal index of $\varrho(\mathscr{A}(\mathcal{O}))$ in $\mathscr{A}(\mathcal{O})$.

As explained in the introduction we shall give two proofs of this theorem. We give now a first proof of Theorem 7.1 by assuming the existence of a field net $\mathcal{O} \rightarrow \mathscr{F}(\mathcal{O})$ of von Neumann algebras, a compact group $G$ (the gauge group) and an action $\alpha: G \rightarrow \operatorname{Aut}(\mathscr{F})$, where $\mathscr{F}=\bigcup_{\mathcal{O} \in \mathscr{K}} \mathscr{F}(\mathcal{O})^{-}$, such that

$$
\alpha_{g}(\mathscr{F}(\mathcal{O}))=\mathscr{F}(\mathcal{O}), \quad g \in G .
$$

$\mathscr{A}(\mathcal{O})$ is the $\alpha$-fixed point algebra of $\mathscr{F}(\mathcal{O})$,

$$
\mathscr{A}(\mathcal{O})=\mathscr{F}(\mathcal{O})^{\alpha},
$$

and there exists an element $g_{0}$ in the center of $G$ with $g_{0}^{2}=$ id such that Bose and Fermi fields

$$
\mathscr{F}_{ \pm}=\left\{T \in \mathscr{F} \mid \alpha_{g_{0}}(T)= \pm T\right\}
$$

localized in space-like separated regions commute or anticommute according to one of them is a Bose field or borh of them are Fermi fields.

If $H \in \mathscr{H}^{\alpha}(\mathscr{F}(\mathcal{O}))$ is a $\alpha$-invariant Hilbert space then the inner endomorphism $\varrho_{H}$ implemented by $H$ on $\mathscr{F}$ restricts to $\mathscr{A}$ to an endomorphism $\left.\varrho_{H}\right|_{\mathscr{A}} \in \mathscr{E}$, localized in $\mathcal{O}$ and we assume that all elements in $\mathscr{E}_{c}$ arise in this way.

We further assume that $\alpha$ is a prime action, namely

$$
\mathscr{A}(\mathcal{O})^{\prime} \cap \mathscr{F}(\mathcal{O})=\mathbf{C}, \quad \mathcal{O} \in \mathscr{K}
$$

that implies that $\alpha \mid \mathscr{F}(\mathcal{O})$ is dominant because $\mathscr{A}(\mathcal{O})$ is properly infinite [25].

This structure has been recently announced by Doplicher and Roberts to follow from general assumptions of Quantum Field Theory [9].

Lemma 7.2. a) If $\phi$ is a left inverse of $\varrho \in \mathscr{E}_{c}$, then

$$
\varepsilon_{\phi} \equiv \varrho \cdot \phi
$$

is a conditional expectation of $\mathscr{A}$ onto $\varrho(\mathscr{A})$ and $\varepsilon_{\phi}(\mathscr{A}(\mathcal{O}))=\varrho(\mathscr{A}(\mathcal{O}))$ if $\mathcal{O} \supset \mathcal{O}_{\varrho}, \mathcal{O} \in \mathscr{K}$.

Conversely if $\varepsilon$ is a conditional expectation of $\mathscr{A}$ onto $\varrho(\mathscr{A})$ then

$$
\phi_{\varepsilon} \equiv \varrho^{-1} \cdot \varepsilon
$$

is a left inverse for $\varrho$. 
b) If $d(\varrho)<\infty$ then every left inverse of $\varrho$, thus every conditional expectation of $\mathscr{A}$ onto $\varrho(\mathscr{A})$, is automatically locally normal.

Proof. a) Clearly $\phi$ maps $\mathscr{A}(\mathcal{O})$ onto $\varrho(\mathscr{A}(\mathcal{O}))$ and $\varrho(\mathscr{A}(\mathcal{O})) \subset \mathscr{A}(\mathcal{O})$ if $\mathcal{O} \supset \mathcal{O}_{\varrho}$. If $T \in \mathscr{A}(\mathcal{O})$, then

$$
\varepsilon_{\phi}(\varrho(T))=\varrho \cdot \phi \cdot \varrho(T)=\varrho(T),
$$

thus $\varepsilon_{\phi} \mid \varrho(\mathscr{A})=$ id. Since $\phi$ is completely positive and unital, $\phi$ has norm 1 , hence $\varepsilon_{\phi}$ has norm 1 and is a conditional expectation. The rest is immediate.

b) This follows by a) and Proposition 5.7 and the following inequality (7.5).

First proof of Theorem 7.1. Let $\varrho \in \mathscr{E}_{c}$ be the restriction to $\mathscr{A}$ of the inner endomorphism $\varrho_{H}$ of $\mathscr{F}$ implemented by the $\alpha$-invariant Hilbert space $H \in \mathscr{H}^{\alpha}(\mathscr{F}(\mathcal{O}))$, where $\mathcal{O} \in \mathscr{K}$ as above.

By Corollary 6.10

$$
\operatorname{Ind}\left(\varrho(\mathscr{A}(\mathcal{O}), \mathscr{A}(\mathcal{O}))=(\operatorname{dim} H)^{2} .\right.
$$

We may assume $\operatorname{dim} H<\infty$ (the argument that follows will also cover the case $\operatorname{dim} H$ infinite).

Let $\varepsilon$ be the conditional expectation of $\mathscr{F}$ onto $\varrho_{H}(\mathscr{F})$ such that $\varepsilon \mid\left(\varrho_{H}(\mathscr{F})^{\prime} \cap \mathscr{F}\right)$ is the trace of $(H, H)$ (one has a tensor product decomposition $\mathscr{F} \simeq \varrho_{H}(\mathscr{F}) \otimes(H, H)$ ). Then $\varepsilon \mid \mathscr{F}(\mathcal{O})$ is the minimal expectation in $C\left(\mathscr{F}(\mathcal{O}), \varrho_{H}(\mathscr{F}(\mathcal{O}))\right.$. Now $\varepsilon_{0} \equiv \varepsilon \mid \mathscr{A}$ is a conditional expectation of $\mathscr{A}$ onto $\varrho(\mathscr{A})$ and by Theorem 6.3 and Corollary 6.10 $\varepsilon_{0}$ is the minimal expectation in $C\left(\mathscr{A}(\mathcal{O}), \varrho(\mathscr{A}(\mathcal{O}))\right.$. By Lemma $7.2 \phi \equiv \varrho^{-1} \cdot \varepsilon_{0}$ is a left inverse of $\varrho$ and we have to show that $\phi$ is the standard left inverse of $\varrho$ and $d(\varrho)=\operatorname{dim} H$.

By definition $\phi=\varrho^{-1} \cdot \varepsilon_{0}$ thus, by the previous notations,

$$
\lambda_{\varrho}=\phi\left(\epsilon_{\varrho}\right)=\varrho^{-1}\left(\varepsilon_{0}\left(\epsilon_{\varrho}\right)\right) \text {, }
$$

and we have to prove that the square of

$$
\varepsilon\left(\epsilon_{\varrho}\right)=\varepsilon_{0}\left(\epsilon_{\varrho}\right)=\lambda_{\varrho}
$$

is a scalar and $\left|\lambda_{\rho}\right|=(\operatorname{dim} H)^{-1}$.

Let $\left\{v_{i}, i \in I\right\}$ be a basis of $H$ that we may choose so that $v_{i} \in \mathscr{F}_{+}, i \in I_{+}$and $v_{i} \in \mathscr{F}_{-}, i \in I_{-}$, where $I_{+} \cup I_{-}=I$.

Let $K$ be Hilbert space in $\mathscr{H}^{\alpha}\left(\mathscr{F}\left(\mathcal{O}_{1}\right)\right)$, with $\mathcal{O}_{1} \in \mathscr{K}$ space-like separated to $\mathcal{O}$ such that $\alpha \mid K$ is equivalent to $\alpha \mid H$ and let $\left\{w_{i}, i \in I\right\}$ be a basis of $K$ that corresponds to $\left\{v_{i}, i \in I\right\}$ under the unitary equivalence.

The space

$$
(K, H)=\text { lin. space }\left\{w v^{*}, w \in K, v \in H\right\}
$$

is $\alpha$ invariant and isomorphic to the tensor product of $K$ and $H^{*}$. The restriction $\alpha \mid(K, H)$ is equivalent to $\pi \otimes \bar{\pi}$, where $\pi=\alpha \mid H$ and $\bar{\pi}$ is the conjugate representation of $\pi$. It follows that the unitary

$$
u=\sum_{i \in I} w_{i} v_{i}^{*}=\sum_{i \in I_{+}} w_{i} v_{i}^{*}+\sum_{i \in I_{-}} w_{i} v_{i}^{*}=u_{+}+u_{-}
$$


is $\alpha$-invariant, thus $u$ and $u_{+}, u_{-}$belong to $\mathscr{A}$. Since

$$
\varrho^{\prime} \equiv \varrho_{K}\left|\mathscr{A}=u \varrho_{H} \cdot(\cdot) u^{*}\right| \mathscr{A}=u \varrho(\cdot) u^{*}
$$

is localized space-like to $\varrho$, it remains to check that

$$
\lambda_{\varrho}^{2} \equiv \varepsilon\left(u^{*} \varrho(u)\right)^{2}=(\operatorname{dim} H)^{-2} .
$$

A direct computation (see [27]) shows that $\varepsilon$ is given by

$$
\varepsilon(x)=\frac{1}{n} \sum_{i, j \in I} v_{i} v_{j}^{*} x v_{j} v_{i}^{*}, \quad x \in \mathscr{F},
$$

where $n=\operatorname{dim} H$; hence

$$
\begin{aligned}
\varepsilon(u) & =\frac{1}{n} \sum_{i, j \in I} v_{i} v_{j}^{*} u v_{j} v_{i}^{*}=\frac{1}{n} \sum_{i, j, k \in I} v_{i} v_{j}^{*} w_{k} v_{k}^{*} v_{j} v_{i}^{*} \\
& =\frac{1}{n} \sum_{i, j \in I} v_{i} v_{j}^{*} w_{j} v_{i}^{*}=\frac{1}{n} \sum_{j \in I} \varrho\left(v_{j}^{*} w_{j}\right) \\
& =\frac{1}{n} \varrho\left(\sum_{i \in I_{+}} w_{j} v_{j}^{*}-\sum_{j \in I_{-}} w_{j} v_{j}^{*}\right)=\frac{1}{n} \varrho\left(u_{+}-u_{-}\right),
\end{aligned}
$$

where we have used the normal commutation relations between $v_{j}$ and $w_{j}$.

In conclusion

$$
\begin{aligned}
\varepsilon\left(\epsilon_{\varrho}\right) & =\varepsilon\left(u^{*} \varrho(u)\right)=\varepsilon(u)^{*} \varrho(u)=\frac{1}{n} \varrho\left(u_{+}-u_{-}\right)^{*} \varrho\left(u_{+}+u_{-}\right) \\
& =\frac{1}{n} \varrho\left(u_{+}^{*} u_{+}-u_{-}^{*} u_{-}\right)=\frac{1}{n} \varrho\left(E_{+}-E_{-}\right),
\end{aligned}
$$

where

$$
E_{ \pm}=\sum_{i \in I_{ \pm}} v_{i} v_{i}^{*}
$$

are orthogonal projections with sum 1 and

$$
\varepsilon\left(\epsilon_{\varrho}\right)^{2}=\frac{1}{n^{2}} \varrho\left(\left(E_{+}-E_{-}\right)^{2}\right)=\frac{1}{n^{2}} .
$$

Notice that in the above proof we have implicitly proved the following.

Proposition 7.3. If $\varrho \in \mathscr{E}_{c}$ has finite statistics, a left inverse of $\phi$ of $\varrho$ is the standard left inverse of $\varrho$ iff $\varepsilon_{\phi}=\varrho \cdot \phi$ restricts to the minimal conditional expectation in $C\left(\mathscr{A}(\mathcal{O}), \varrho(\mathscr{A}(\mathcal{O}))\right.$ for some, hence for all, $\mathcal{O} \in \mathscr{K}, \mathcal{O} \supset \mathcal{O}_{\varrho}$.

We shall give a direct proof of Proposition 7.3 in the second proof of Theorem 7.2. This proof will be at the observable level, making no use of the field algebra and the gauge group. We begin with the following.

Lemma 7.4. If $\varrho \in \mathscr{E}_{c}$ is localized in $\mathcal{O}$ then $\varrho(\mathscr{A})^{\prime} \cap \mathscr{A} \subset \varrho(\mathscr{A}(\mathcal{O}))^{\prime} \cap \mathscr{A}(\mathcal{O})$.

Proof. Let $T \in \varrho(\mathscr{A})^{\prime} \cap \mathscr{A}$; since $\varrho \mid \mathscr{A}\left(\mathcal{O}_{1}\right)=$ id if $\mathcal{O}_{1}$ is a spacelike to $\mathcal{O}$, we have 
$T \in \varrho\left(\mathscr{A}\left(\mathcal{O}_{1}\right)\right)^{\prime}=\mathscr{A}\left(\mathcal{O}_{1}\right)^{\prime}$, hence $T \in \mathscr{A}\left(\mathcal{O}^{\prime}\right)^{\prime}=\mathscr{A}(\mathcal{O})$ by duality. Hence $T \in \varrho(\mathscr{A})^{\prime} \cap$ $\mathscr{A}(\mathcal{O}) \subset \varrho(\mathscr{A}(\mathcal{O}))^{\prime} \cap \mathscr{A}(\mathcal{O})$.

In the second proof of Theorem 7.2 we shall consider for simplicity only elements $\varrho \in \mathscr{E}_{c}$ with finite statistics and shall prove that

$$
\operatorname{Ind}_{\varepsilon}[\varrho(\mathscr{A}(\mathcal{O})), \mathscr{A}(\mathcal{O})]=d(\varrho)^{2},
$$

where $\varepsilon \in C\left(\mathscr{A}(\mathcal{O}), \varrho(\mathscr{A}(\mathcal{O}))\right.$ is the restriction to $\mathscr{A}(\mathcal{O})$ of $\varepsilon_{\phi}=\varrho \cdot \phi$, where $\phi$ is the unique left inverse of $\varrho$.

The statement of Theorem 7.2 that $d(\varrho)$ equals the minimal index of $\varrho(\mathscr{A}(\mathcal{O}))$ in $\mathscr{A}(\mathcal{O})$ can be obtained by further assuming that

$$
\varrho(\mathscr{A}(\mathcal{O}))^{\prime} \cap \mathscr{A}(\mathcal{O})=\mathbf{C}
$$

whenever $\varrho$ is irreducible and localized in $\mathcal{O} \in \mathscr{K}$.

We shall comment about the validity of the relation (7.3) in Proposition 7.5.

Second proof of Theorem 7.2. We begin by assuming that $\varrho$ is irreducible. In this case Lemma 3.8 of [7] shows that

$$
\left\|\phi\left(T^{*} T\right)\right\| \geqq \lambda_{\varrho}^{2}\left\|T^{*} T\right\|, \quad T \in \mathscr{A} .
$$

Since $\phi=\varrho^{-1} \cdot \varepsilon_{\phi}$ by Lemma 7.1 and $\varrho$ is isometric we have

$$
\left\|\varepsilon_{\phi}\left(T^{*} T\right)\right\| \geqq \lambda_{\varrho}^{2}\left\|T^{*} T\right\|, \quad T \in \mathscr{A},
$$

hence by Theorem 4.1

$$
\text { Ind }[\varrho(\mathscr{A}(\mathcal{O})), \mathscr{A}(\mathcal{O})] \leqq \lambda_{\varrho}^{-2}=d(\varrho)^{2} .
$$

By Theorem 4.1 we have to show that $\left|\lambda_{e}\right|$ is the best constant such that $\left\|\varepsilon_{\phi}(T)\right\| \geqq \lambda_{\varrho}^{2}\|T\|, T \in \mathscr{A}(\mathcal{O})^{+}$, or equivalently such that

$$
\|\phi(T)\| \geqq \lambda_{\varrho}^{2}\|T\|, \quad T \in \mathscr{A}(\mathcal{O})^{+} .
$$

Let $\epsilon_{o}^{n}$ be the representation of the permutation group $\mathbf{P}_{n}$ associated with $\varrho$ [7], $n \in \mathbf{N}$, and $E_{ \pm}^{n}$ the totally symmetric or anti-symmetric projection in the group algebra $\mathbf{C}\left[\mathbf{P}_{n}\right]$. Then by [7, Lemma 5.1] with $F_{ \pm}^{n} \equiv \epsilon_{\rho}^{n}\left(E_{ \pm}^{n}\right)$, one has

$$
\phi\left(F_{+}^{n}\right)=\lambda_{\varrho}^{2} F_{+}^{n-1} \text { or } \phi\left(F_{-}^{n}\right)=\lambda_{\varrho}^{2} F_{-}^{n-1},
$$

where $n=d(\varrho)$, according to whether $\lambda_{e}= \pm 1 / n$. It follows that

$$
\left\|\phi\left(F_{*}^{n}\right)\right\|=\lambda_{\varrho}^{2}\left\|F_{*}^{n-1}\right\|=\lambda_{\varrho}^{2},
$$

where $*=+$ or - and $\lambda_{\varrho}^{2}$ is the desired best constant.

It remains to consider the case where $\varrho$ is reducible. We apply [7, Proposition 6.6] and assume $d(\varrho)<\infty$ so that $\varrho(\mathscr{A})^{\prime} \cap \mathscr{A}$ is finite dimensional. If $\phi$ is the standard left inverse of $\varrho$ and $\varepsilon_{\phi}=\varrho \cdot \phi$, then

$$
\varepsilon_{\phi}\left|\varrho(\mathscr{A})^{\prime} \cap \mathscr{A}=\phi\right| \varrho(\mathscr{A})^{\prime} \cap \mathscr{A}=\operatorname{Tr}(\cdot),
$$

where $\operatorname{Tr}$ is a faithful tracial state. Let $E_{i}$ be a partition of the unit by minimal projections of $\varrho(\mathscr{A})^{\prime} \cap \mathscr{A}$. Then $T \rightarrow \varrho(T) E_{i}$ is an irreducible representation of $\mathscr{A}$ that corresponds to a $\varrho_{i} \in \mathscr{E}_{c}$ and

$$
d\left(\varrho_{i}\right)=\operatorname{Tr}\left(E_{i}\right) d(\varrho) .
$$

Setting $I_{i}=\operatorname{Ind}\left[\varrho_{i}(\mathscr{A}(\mathcal{O})), \mathscr{A}(\mathcal{O})\right]$ the above discussion shows that 


$$
I_{i}^{1 / 2}=\operatorname{Ind}\left[\varrho_{i}(\mathscr{A}(\mathcal{O})), \mathscr{A}(\mathcal{O})\right]^{1 / 2}=\operatorname{Ind}\left[\varrho(\mathscr{A}(\mathcal{O})) E_{i}, \mathscr{A}(\mathcal{O}) E_{i}\right]^{1 / 2}=d\left(\varrho_{i}\right)=\operatorname{Tr}\left(E_{i}\right) d(\varrho),
$$

where we have used Lemma 7.4 that entails that $E_{i} \in \varrho(\mathscr{A}(\mathcal{O}))^{\prime} \cap \mathscr{A}(\mathcal{O})$ and $\varrho_{i}$ is localized in $\mathcal{O}$. It follows by Theorem 5.5 that

$$
\text { Ind }[\varrho(\mathscr{A}(\mathcal{O})), \mathscr{A}(\mathcal{O})]^{1 / 2}=\sum_{i} I_{i}^{1 / 2}=d(\varrho) \text {. }
$$

We now consider a property that will improve Lemma 7.4. Denote by $M \vee N$ the von Neumann algebra generated by $M$ and $N$. If $\mathscr{O} \in \mathscr{K}$ let $\mathscr{B}_{\mathscr{O}}$ be the $C^{*}$-algebra generated by the union $\mathscr{A}(\mathcal{O}) \vee \mathscr{A}\left(\mathcal{O}_{1}\right), \mathcal{O}_{1} \subset \mathcal{O}^{\prime}, \mathcal{O}$ bounded. By duality $\mathscr{B}_{\mathscr{O}}$ is irreducible.

Proposition 7.5. Let $\varrho \in \mathscr{E}_{\mathrm{c}}$ be localized in $\mathcal{O}$. If $d(\varrho)<2$, then

$$
\varrho(\mathscr{A}(\mathcal{O}))^{\prime} \cap \mathscr{A}(\mathcal{O})=\mathbf{C} .
$$

In general the above relation is equivalent to the irreducibility of $\left.\varrho\right|_{\mathscr{B}}$.

Proof. If $d(\varrho)<2$, the above proof shows that Ind $[\varrho(\mathscr{A}(\mathcal{O}), \mathscr{A}(\mathcal{O})]<4$, hence $(7.3)$ automatically holds e.g. by Theorem 5.5 because the index is always greater or equal to 1 . In general if $\varrho\left(\mathscr{B}_{\mathscr{O}}\right)$ is irreducible we will show the reverse inclusion in Lemma 7.4. Let $T \in \varrho(\mathscr{A}(\mathcal{O}))^{\prime} \cap \mathscr{A}(\mathcal{O})$, thus in particular $T \in \mathscr{A}\left(\mathcal{O}_{1}\right)^{\prime}$ if $\mathcal{O}_{1} \subset \mathcal{O}^{\prime}$ and, since $\varrho$ is localized in $\mathcal{O}, T \in \varrho\left(\mathscr{A}\left(\mathcal{O}_{1}\right)\right)^{\prime}$. Therefore

$$
T \in \varrho(\mathscr{A}(\mathcal{O}))^{\prime} \cap \varrho\left(\mathscr{A}\left(\mathcal{O}_{1}\right)\right)^{\prime}=\left[\varrho(\mathscr{A}(\mathcal{O})) \vee \varrho\left(\mathscr{A}\left(\mathcal{O}_{1}\right)\right)\right]^{\prime}=\varrho\left(\mathscr{A}(\mathcal{O}) \vee \mathscr{A}\left(\mathcal{O}_{1}\right)\right)^{\prime},
$$

and by assumptions $T \in \varrho\left(\mathscr{B}_{\mathscr{O}}\right)^{\prime}=\mathbf{C}$. The rest is now clear.

\section{The Statistics of Low Dimensional Theories}

As mentioned in the introduction the results in Sect. 7 are valid under the assumption that the Minkowski space $\mathbf{R}^{d}$ has dimension $d>2$. When $d=2$ the structure is more complicated and exotic statistics arise.

In this section we assume $d=2$ and will extend Theorem 7.1 to this case by a further analysis of the second proof of that Theorem. Note that the correspondence between superselection sectors and localized endomorphisms is also valid in particular in a conformal theory [5].

While the inequality corresponding to (7.4) will put an a priori bound on the index of a sector, for the exact evaluation of the index we shall need the existence of a conjugate sector; this assumption is rather general and has been discussed in $[8$, appendix] and in [12].

Since our proof will rely on lemmas that are natural adaptations of arguments in $[7,8]$ we shall be sketchy in the sequel and indicate the points where the argument is extended. ${ }^{2}$

We use the assumptions and the notations of Sect. 7.

If $\mathcal{O} \subset \mathbf{R}^{2}, \mathcal{O} \in \mathscr{K}$, is a double cone, its space-like complement $\mathcal{O}^{\prime}$ has two connected components. We shall say that $\mathcal{O}_{1} \subset \mathbf{R}^{2}$ is left (right) space-like to $\mathscr{O}$ if $\mathcal{O}_{1}$ lies in the left (right) connected component of $\mathcal{O}^{\prime}$.

\footnotetext{
${ }^{2}$ While this paper was being typed, we received a preliminary form of [11] that contains in particular a more complete description of some lemmas then in this section
} 
With $\varrho \in \mathscr{E}_{c}$ a localized endomorphism of $\mathscr{A}=\cup \mathscr{A}(\mathcal{O})^{-}$, we choose a unitary $u \in \mathscr{A}$ such that $\varrho^{\prime} \equiv u \varrho(\cdot) u^{*} \in \mathscr{E}_{c}$ is localized left spacelike to $\varrho$ and put

$$
\epsilon_{\varrho} \equiv u^{*} \varrho(u) \text {. }
$$

As before $\epsilon_{\varrho}$ is independent of $u$. If $u$ had been chosen so that $\varrho^{\prime}$ were localized right space-like to $\varrho$, then (8.1) would have given another unitary $\epsilon_{\varrho}^{*}$. An elementary but basic observation is the following.

Lemma 8.1. $\epsilon_{\varrho}^{*}$ is the adjoint of $\epsilon_{\varrho}$.

Proof. Let $\varrho_{i} \equiv u_{i} \varrho(\cdot) u_{i}^{\prime} \in \mathscr{E}_{c}$ with $u_{i}$ unitaries of $\mathscr{A}(i=1,2)$ such that $\varrho_{1}$ and $\varrho_{2}$ are localized respectively left and right space-like to $\varrho$. As in [7, Lemma 2.6] the unitary

$$
v \equiv \varrho\left(u_{2}\right)^{*} u_{1}^{*} u_{2} \varrho\left(u_{1}\right)
$$

is independent of $u_{1}, u_{2}$. Setting $u_{2}=1$ we see that $v=\epsilon_{\varrho}$. Setting $u_{1}=1$ we see that $v^{*}=\epsilon_{\varrho}^{*}$.

Following the argument in [7, Lemma 3.3] we may construct a left inverse $\psi$ of $\varrho \in \mathscr{E}_{c}$. If $\varrho$ is irreducible $\psi\left(\epsilon_{\varrho}\right) \in \mathbf{C}$. In general we may consider a standard left inverse, namely a left inverse $\psi$ such that

$$
\lambda_{\varrho}=\psi\left(\epsilon_{\varrho}\right)
$$

is a multiple of a unitary (the argument in [7, Proposition 6.3] gives the existence of a standard left inverse, unique if $\psi\left(\epsilon_{\varrho}\right) \neq 0$ for some left inverse $\varrho$, but we shall not need this fact here).

In this case $\lambda_{\varrho}^{*} \lambda_{\varrho}$ is a scalar and we may define the statistical dimension of $\varrho$ by

$$
d(\varrho)=\left|\lambda_{\varrho}\right|^{-1} \text {. }
$$

Lemma 8.2. If $\varrho_{1}, \varrho_{2} \in \mathscr{E}_{c}$ are irreducible with left inverse $\psi_{1}, \psi_{2}$ then $\psi_{2} \psi_{1}$ is a standard left inverse of $\varrho_{1} \varrho_{2}$ and

$$
d\left(\varrho_{1} \varrho_{2}\right)=d\left(\varrho_{1}\right) d\left(\varrho_{2}\right) .
$$

Proof. This lemma is proved by an extension of the argument in [7, Lemma 6.7], see also [11].

Lemma 8.3. Let $\varrho \in \mathscr{E}_{c}$ be an endomorphism with a standard left inverse $\psi$. If $E$ is $a$ minimal projection of $\varrho(\mathscr{A})^{\prime} \cap \mathscr{A}$ such that the reduction of $\varrho$ by $E$ is equivalent to the identity representation of $\mathscr{A}$, then

$$
d(\varrho)=\psi(E)^{-1} .
$$

Proof. The proof is obtained by applying [7, Proposition 6.5] to the special case where $\left.\varrho\right|_{E}$ is equivalent to the identity id, because $\epsilon_{\mathrm{id}}=1$.

Recall that an endomorphism $\varrho \in \mathscr{E}_{c}$ is conjugate to $\varrho$ if $\varrho \varrho$ contains the identity subrepresentation. If $\varrho \in \mathscr{E}_{c}$ is irreducible and $\bar{\varrho}$ exists, then $\varrho$ can be chosen irreducible and $\varrho \varrho \varrho$ contains the identity subrepresentation with multiplicity 1 [8].

Lemma 8.4. Let $\varrho, \varrho \in \mathscr{E}_{c}$ be irreducible conjugate endomorphisms. Then

$$
d(\varrho)=d(\bar{\varrho})
$$


Proof. The proof of [8, Theorem 3.1] still applies and shows that $\lambda_{\varrho}=\lambda_{\bar{\varrho}}$.

We now give the desired interpretation of $d(\varrho)$. For simplicity we deal with irreducible sectors. The general case is then a consequence of the additivity of the statistical dimension [7, Proposition 6.6] and the additivity of the square root of the minimal index (Theorem 5.5).

Theorem 8.5. Let $\varrho \in \mathscr{E}_{\mathrm{c}}$ be an irreducible localized endomorphism. If there exists a conjugate endomorphism $\bar{\varrho}$, and $\mathcal{O} \in \mathscr{K}$ is sufficiently large that $\varrho$ and $\bar{\varrho}$ are both localized in $\mathcal{O}$, then

$$
\operatorname{Ind}_{\varepsilon}[\varrho(\mathscr{A}(\mathcal{O})), \mathscr{A}(\mathcal{O})]=d(\varrho)^{2},
$$

where $\varepsilon$ is the restriction to $\mathscr{A}(\mathcal{O})$ of the conditional expectation $\varrho \cdot \psi$ associated with the unique left inverse $\psi$. In particular if $d(\varrho)<\infty$ and the property in Proposition 7.5 holds then $\varepsilon$ is the minimal expectation of $\mathscr{A}(\mathcal{O})$ onto $\varrho(\mathscr{A}(\mathcal{O}))$.

Proof. As mentioned before we may choose $\bar{\varrho} \in \mathscr{E}_{c}$ irreducible so that $\eta \equiv \bar{\varrho} \varrho \in \mathscr{E}_{c}$ contains the identity representation with multiplicity 1 . Let $E \in \eta(\mathscr{A})^{\prime} \cap \mathscr{A}$ be the minimal projection corresponding to the identity subrepresentation and note that $E \in \eta(\mathscr{A}(\mathcal{O}))^{\prime} \cap \mathscr{A}(\mathcal{O})$ by Lemma 7.4. With $\bar{\psi}$ the left inverse of $\bar{\varrho}, \psi \bar{\psi}$ is a standard left inverse of $\varrho \varrho$ by Lemma 8.2 and by Lemma 8.4,

$$
d(\eta)=d(\varrho) d(\bar{\varrho})=d(\varrho)^{2} .
$$

By applying Lemma 8.3 we have

$$
d(\varrho)^{-2}=d(\eta)^{-1}=\psi \bar{\psi}(E) .
$$

As in Sect. 7 we have

$$
\|\psi(T)\| \geqq\left|\lambda_{\varrho}\right|\|T\|, \quad T \in \mathscr{A}^{+},
$$

and we have to show that $\left|\lambda_{e}\right|$ is the best constant in this inequality and then apply Theorem 4.1.

Now $E \in \eta(\mathscr{A})^{\prime} \cap \mathscr{A}$, thus

$$
\varrho(\varrho(T)) E=E \bar{\varrho}(\varrho(T)), \quad T \in \mathscr{A},
$$

and if we evaluate $\bar{\psi}$ on both sides of this inequality we have

$$
\varrho(T) \bar{\psi}(E)=\bar{\psi}(E) \varrho(T), \quad T \in \mathscr{A},
$$

namely $\bar{\psi}(E) \in \varrho(\mathscr{A})^{\prime} \cap \mathscr{A}=\mathbf{C}$.

It follows that

$$
\psi(E)=\psi \bar{\psi}(E)=\left|\lambda_{\eta}\right|=\left|\lambda_{\rho}\right|^{2},
$$

showing that $\left|\lambda_{\varrho}\right|^{2}$ is the optimal constant in (8.2).

Corollary 8.6. If $\varrho \in \mathscr{E}_{c}$ is the direct sum of irreducible sectors all having a conjugate sector then

$$
d(\varrho) \in\left\{2 \cos \frac{\pi}{n}, n=3,4, \ldots\right\} \cup[2, \infty] .
$$

Proof. Immediate by Corollary 4.3 and Theorem 8.5 . 


\section{Outlook}

This work bring up the need for further analysis. We partly mention here some developments connected with the statistics problem.

First of all one should pass from the identification of the index of $\varrho(\mathscr{A}(\mathcal{O})) \subset \mathscr{A}(\mathcal{O})$ to the analysis of the "higher order" information contained in this inclusion. On one hand this amounts to the computation of the Jones tower [19] or of the tower $\gamma^{n}(\mathscr{A}(\mathcal{O}))$, where $\gamma: \mathscr{A}(\mathcal{O}) \rightarrow \varrho(\mathscr{A}(\mathcal{O}))$ is a canonical endomorphism [22], and to the identification of the Ocneanu graph invariant [28].

On the other hand one has to study the braid group representations giving the statistics (a first analysis is now contained in [11]) and relate it to the tower structure.

The paragroup arising in this way [28] should be related to an extended "gauge group." For example if the superselection structure is singly generated, most if not all the information on the gauge group are to be contained in the tower associated with the generator and in the braid group representation.

Further a priori restrictions on the values of the statistical dimension are tied up with the existing problem on the values of the index for subfactors with trivial relative commutant of the hyperfinite $I_{1}$-factor [19]. This is a point where the algebraic structure of the single local algebra enters. By considering for example a wedge region, we know that the associated observable von Neumann algebra is a factor of type $I I I_{1}$ and (assuming the split property) injective, see [25, 3]. Our analysis by Takesaki duality then reduces to the consideration of the injective factor of type $I I_{1}$.

Another relevant point would be to describe the rather apparent relation between the statistical dimension and the central charge in two dimensional conformal field theory, cf. [13]; this would confirm the general feeling of an existing relation of the central charge with the Jones projections, see [20].

Finally we mention a widespread hope that the analysis of low dimensional statistics might lead to a better understanding of low dimensional critical phenomena as high temperature superconductivity and fractional Hall effect.

\section{References}

1. Araki, H., Haag, R., Kastler, D., Takesaki, M.: Extension of KMS states and chemical potential. Commun. Math. Phys. 53, 97-134 (1977)

2. Bisognano, J. J., Wichmann, E. H.: On the duality for a hermitian scalar field J. Math. Phys. 16, 985-1007 (1975)

3. Buchholz, D., D'Antoni, C., Fredenhagen, K.: The universal structure of local algebras. Commun. Math. Phys. 111, 123 (1987)

4. Buchholz, D., Fredenhagen, K.: Locality and the structure of particle states. Commun. Math. Phys. 84, 1-54 (1982)

5. Buchholz, D., Mack, G., Todorov, I.: The current algebra on the circle as a germ of local field theories. preprint

6. Connes, A.: Une classification des facteurs de type III. Ann. Sci. Ec. Norm. Sup. 8, 383-419 (1975)

7. Doplicher, S., Haag, R., Roberts, J. E.: Local observables and particle statistics I. Commun. Math. Phys. 23, 199-230 (1971)

8. Doplicher, S., Haag, R., Roberts, J. E.: Local observables and particle statistics II. Commun. Math. Phys. 35, 49-85 (1974)

9. Doplicher, S., Roberts, J. E.: Why there is a field algebra in preparation

10. Doplicher, S., Longo, R.: Standard and split inclusions of von Neumann algebras. Invent. Math. 75, 493-536 (1984) 
11. Fredenhagen, K., Rehren, K. H., Schroer, B.: Superselection sectors with braid group statistics and exchange algebras, I: General theory, manuscript

12. Fredenhagen, K.: On the existence of antiparticles. Commun. Math. Phys. 79, 141-151 (1981)

13. Friedan, D., Qui, Z., Shenker, S.: Conformal invariance, unitarity, and two dimensional critical exponents. In: Vertex operators in mathematics and in physics. Lepowsky, J., Mandelstam, S., Singer, I. M. (eds.). Berlin, Heidelberg, New York: Springer 1984

14. Fröhlich, J.: Statistics of fields, the Yang-Baxter equation, and the theory of knots and links, preprint

15. Greenberg, O. W., Messiah, A. M. L.: Symmetrization postulate and its experimental formulation. Phys. Rev. 136, B 248-267 (1964)

16. Hamachi, T., Kosaki, H.: Index and flow of the weights of factors of type III, manuscript

17. Hiai, F.: Minimizing indices of conditional expectations onto subfactors, preprint

18. Kosaki, H.: Extension of Jones' theory on index to arbitrary factors. J. Funct. Anal. 66, 123-140 (1986)

19. Jones, V. F. R.: Index for subfactors. Invent. Math. 72, 1-25 (1983)

20. Jones, V. F. R.: Subfactors and related topics, preprint

21. Longo, R.: Solution of the factorial Stone-Weirstrass conjecture. Invent. Math. 76, 145-155 (1984)

22. Longo, R.: Simple injective subfactors. Adv. Math. 63, 152-171 (1987)

23. Longo, R.: The joint modular structure of an inclusion of von Neumann algebras. Contemp. Math. 62, 529-538 (1987)

24. Longo, R.: Restricting a compact action to an injective subfactors. Ergod. Th. Dynam. Syst. 9, 127-135 (1989)

25. Longo, R.: Algebraic and modular structure of von Neumann algebras of Physics. Proc. Sympos. Pure Math. 38, 551-566 (1982)

26. Longo, R.: Inclusions of von Neumann algebras and Quantum Field Theory. In: IXth International Conference on Mathematical Physics. Simon, B., Truman, A., Davies, I. M. (eds.). Swansea, July 1988. Bristol and New York: Adam Hilger

27. Longo, R.: In preparation

28. Ocneanu, A.: Quantized groups, string algebras and Galois theory for algebras, preprint

29. Pimsner, M., Popa, S.: Entropy and index for subfactors. Ann. Sci. Ec. Norm. Sup. 19, 57-106 (1986)

30. Roberts, J. E.: Cross products of von Neumann algebras by group duals. Sympos. Math. 20, 335-363 (1976)

31. Takesaki, M.: Duality for crossed products and the structure of von Neumann algebras of type III. Acta Math. 131, 249-310 (1973)

32. Takesaki, M.: Conditional expectation in von Neumann algebras. J. Funct. Anal. 9, 306-321 (1970)

33. Schroer, B.: New methods and results in conformal $\mathrm{QFT}_{2}$ and the string idea-preprint

34. Baillet, M., Donizeau, Y., Havet, J.F.: Indice d'une esperance conditionelle. Comp. Math. 66, 199-236 (1988)

35. Longo, R.: Index of subfactors and statistics of quantum fields, II: Correspondences, braid group statistics and Jones polynomial-preprint

Communicated by A. Connes

Received November 3, 1988

Note added in proofs. The analysis of the low dimensional statistics according to the lines here proposed proceeds in [35]. The conjugate sector $\bar{\rho}$ is 'described in general by the formula $\bar{\rho}=\rho^{-1} \cdot \gamma$ where $\gamma: \mathscr{A}(\mathcal{O}) \rightarrow \mathscr{A}(\mathcal{O})$ is a canonical endomorphism, so that the corresponding tower is $\mathscr{A}(\mathcal{O}) \supset \rho(\mathscr{A}(\mathcal{O})) \supset$ $\rho \bar{\rho}(\mathscr{A}(\mathcal{O})) \supset \rho \bar{\rho} \rho(\mathscr{A}(\mathcal{O})) \supset \cdots$. In the case of a selfconjugate sector $\rho$ such that $\rho^{2}$ has two irreducible components the field theoretical braid group representation is identified with a Jones representation. In particular the Jones link invariant polynomial $V_{L}$ is explicitly attached to the sector:

$$
V_{L}(q)=(-d(\rho))^{n-1}\left(-p h\left(\lambda_{\rho}\right)\right)^{-l} \phi^{\infty}\left(\epsilon_{\rho}^{(n)}(\alpha)\right)
$$

where $\epsilon_{\rho}^{(n)}$ is the field theoretical representation of the braid group $B_{n}$ determined by $\epsilon_{\rho}^{(n)}\left(\sigma_{i}\right) \equiv \rho^{i-1}\left(\epsilon_{\rho}\right)$.

The assumption on the existence of $\bar{\rho}$ in Theorem 8.5 is unnecessary, since it may be constructed by the Stinespring dilation of $\phi$ by applying Lemma 7.2, b.

Related facts are contained in the final version of [11] (to appear in Commun. Math. Phys.). 
